\title{
Cross-Layer Aided Energy-Efficient Opportunistic Routing in Ad Hoc Networks
}

\author{
Jing Zuo, Chen Dong, Hung Viet Nguyen, Soon Xin Ng, Lie-Liang Yang, and Lajos Hanzo
}

\begin{abstract}
Most of the nodes in ad hoc networks rely on batteries, which requires energy saving. Hence, numerous energyefficient routing algorithms have been proposed for solving this problem. In this paper, we exploit the benefits of cross-layer information exchange, such as the knowledge of the Frame Error Rate (FER) in the physical layer, the maximum number of retransmissions in the Medium Access Control (MAC) layer and the number of relays in the network layer. Energy-consumptionbased Objective Functions (OF) are invoked for calculating the end-to-end energy consumption of each potentially available route for both Traditional Routing (TR) and for our novel Opportunistic Routing (OR), respectively. We also improve the TR and the OR with the aid of efficient Power Allocation (PA) for further reducing the energy consumption. For the TR, we take into account the dependencies amongst the links of a multi-hop route, which facilitates a more accurate performance evaluation than upon assuming the links that are independent. Moreover, two energy-efficient routing algorithms are designed based on Dijkstra's algorithm. The algorithms based on the energy OF provide the theoretical bounds, which are shown to be close to the bound found from exhaustive search, despite the significantly reduced complexity of the former. Finally, the endto-end throughput and the end-to-end delay of this system are analyzed theoretically and a new technique of characterizing the delay distribution of OR is proposed. The simulation results show that our energy-efficient OR outperforms the TR and that their theoretical analysis accurately matches the simulation results.
\end{abstract}

Index Terms-Opportunistic routing, energy-efficient routing, cross-layer, objective function, near-capacity coding, end-to-end throughput, end-to-end delay.

\section{INTRODUCTION}

$\mathbf{E}$ NERGY saving in wireless ad hoc networks is a salient problem, which mitigates the problem of limited battery supply at each node. Numerous energy-efficient algorithms have been proposed for reducing the energy consumption [1$10]$. The authors of $[5,6]$ have aimed for energy saving without considering the specifics of the network layer, the Medium Access Control (MAC) layer or the physical layer. By contrast, the authors of $[1,4,7-13]$ invoked cross-layer optimization, since the energy reduction is related to several layers. The authors of $[2,5]$ conceived energy-efficient routing concepts.

Traditional Routing (TR) relies on a route discovery process invoked for gleaning sufficient routing information for

Manuscript received October 9, 2012; revised March 21, August 9, and October 17, 2013. The editor coordinating the review of this paper and approving it for publication was V. Wong.

The authors are with the School of ECS, University of Southampton, SO17 1BJ, UK (e-mail: \{jz08r, cd2g09, hvn08r, sxn, lly, lh\}@ecs.soton.ac.uk).

The research leading to these results has received funding from the European Union's Seventh Framework Programme (FP7/2012-2014) under grant agreement no 288502. The financial support of the China-UK Scholarship Council, and of the RC-UK under the auspices of the IU-ATC initiative, is also gratefully acknowledged.

Digital Object Identifier 10.1109/TCOMM.2013.121413.120767 the source to make meritorious routing decisions, regardless, whether the routing protocol is proactive or reactive [14]. However, due to the rapid fluctuation of the channel conditions, the routing information estimated on the basis of the average Channel Quality Information (CQI) may become stale, resulting in suboptimum routing. Therefore, Opportunistic Routing (OR) $[2-4,8,11,13,15-20]$ has been proposed for avoiding this problem. In OR no pre-selected route is employed, instead a so-called forwarder relay set is used for forwarding the packets along a beneficial route. The nearinstantaneously varying characteristics of wireless channels is beneficially exploited considered by OR. Liu et al. [16] illustrated the basic idea behind OR and categorized the potential design criteria, including the Estimated Transmission count (ETX), the geographic distance aided and the energy consumption based philosophies. Biswas and Morris [11] proposed an Extremely Opportunistic Routing (ExOR) scheme, which employed the ETX metric at the destination for deciding the priority order of selecting a relay from the potential forwarder set. The proposed routing regime integrated the routing protocol and the MAC protocol for the sake of increasing the attainable throughput of multi-hop wireless networks. Their solution [11] also exploited the less reliable long-distance links, which would have been ignored by traditional routing protocols. Moreover, Dubois-Ferrière et al. [17] conceived the Least-Cost Anypath Routing (LCAR) regime, which finds the optimal choice of candidate relays relying on the expected ETX cost of forwarding a packet to the destination. This LCAR algorithm considers the coordination of the link layer protocols. Laufer et al. [20] proposed a 'polynomial-time multirate anypath' routing algorithm and provided the proof of its optimality. The proposed routing algorithm employed the Expected Anypath Transmission Time (EATT) as the routing metric, which is a generalization of the unidirectional ETX metric that takes into account that nodes transmit at multiple bit rates. The authors of $[2,15,19]$ employed a geographic distance based metric for choosing the potential forwarder relay set. More specifically, Zorzi and Rao [15] proposed an OR scheme based on random forwarding, where the specific node, which is closest to the Destination (D) is chosen as the Relay (R) for the next hop. This paper theoretically analyzed the achievable multi-hop performance. Furthermore, Zorzi and Rao [2] analyzed the achievable energy as well as latency performance and provided a detailed description of a MAC scheme based on both opportunistic concepts and on collision avoidance. Zeng et al. [19] proposed a multirate OR by incorporating rate adaptation into their candidateselection algorithm, which was shown to achieve a higher throughput and lower delay than the corresponding traditional 
single-rate routing and its opportunistic single-rate routing counterpart. The authors of $[3,4,8]$ employed the energy consumption metric for choosing the potential forwarder relay set. More concretely, Mao et al. [3] presented an energyefficient OR strategy relying on sophisticated power allocation, which prioritizes the forwarder relays by directly minimizing the total energy consumption of all nodes. Dehghan et al. [4] developed a minimum-energy cooperative routing based on many-to-many cooperation and determines the optimal route with the aid of the Bellman-Ford algorithm [21]. Wei et al. [8] proposed an energy-conserving Assistant Opportunistic Routing (AsOR) protocol, which classified a sequence of nodes into three different node sets, namely, the frame node, the assistant node and the unselected node. The frame nodes were indispensable for decode-and-forward operation, while the assistant nodes provided protection against unsuccessful opportunistic transmissions. Although the authors of $[3,4,8]$ employed the energy consumption as their routing metric, they have not provided any theoretical bounds in their performance analysis. Moreover, these authors assumed that the number of affordable MAC retransmissions was infinite. Against this background, our novel contributions are:

- Two accurate energy-consumption-based OFs are constructed, which are used for the TR and the OR respectively. We exploit the knowledge of both the Frame Error Ratio (FER) within the physical layer, and of the number of MAC retransmissions as well as of the number of relays in the network layer.

- A routing algorithm is designed for the TR, which employs our energy-consumption-based OF. Similarly, a routing algorithm is designed for OR, which employs our energy-consumption-based OF for ordering the relays (from small to large) in the forwarder set. Theoretical bounds are derived for the Normalized Energy Consumption (NEC) of both the algorithms, which are shown to be close to the ultimate bound obtained with the aid of an exhaustive search.

- The achievable end-to-end throughput, the end-to-end delay and the delay distribution of the system are also evaluated theoretically.

The rest of the paper is organized as follows. Section II theoretically analyzes the performance of the system for the single-hop route, for the TR and for the OR. Section III describes our energy-efficient routing algorithms conceived for TR and OR, respectively. The delay distribution of OR is also analyzed. Finally, Section IV analyzes the overall performance of the system, while Section V provides our conclusions.

\section{TheORETICAL ANALYSis}

In this paper, the transmit energy consumed by the data packets during their transmission is considered under the idealized simplifying assumption that the energy dissipated by other packets, such as the routing and MAC control packets, is negligible. Before defining the proposed energy-consumptionbased OF, the symbols used are defined.

- $H$ : the number of hops in an established route;

- $P_{t_{i}}$ : the transmit power in the $i$-th node of the established route;
- FER $R_{i}$ : the FER of the $i$-th link in an established route;

- $p_{i}$ : the successful probability of the $i$-th link, where $p_{i}=$ $1-F E R_{i}$

- $N_{r}$ : the maximum number of MAC retransmissions, including the first transmission attempt;

- $E_{\text {total }}$ : the total energy consumption;

- $\bar{E}_{\text {total }}$ :

\section{A. FER and power allocation in a single-hop route}

In our previous work [22], an accurate energy-consumptionbased OF was employed for estimating the normalized endto-end energy consumption for a given route under the assumption that the FER, the maximum number of MAC retransmissions and the number of hops were known. Although the energy-consumption-based OF representing the real-world scenarios is beneficial, the resultant best route still wastes energy, since the distances between the different pairs of relays are different. For the sake of ensuring that the total powerconsumption is minimized, each node's transmit power should be different. Therefore, we invoke power control for further reducing the energy consumption by finding the optimum transmit power for each node.

Naturally, the channel conditions, the thermal noise level and the distance between the transmitter and the receiver jointly determine the FER of a link. We conducted simulations for characterizing the FER performance versus the Signalto-Noise-Ratio (SNR) and followed the approach of [12] for fitting a polynomial to the FER versus SNR curve. The Forward Error Correction (FEC) scheme employed in our paper is an Irregular Convolutional Coded, Unity-Rate Coded and Quadrature Phase-Shift Keying (IrCC-URC-QPSK) [23, 24] scheme. The corresponding FER versus SNR curve was generated with the aid of bit-by-bit simulations. The overall FEC code rate was $R_{c}=0.5$, the effective throughput was 1 bps (bits/symbol), the frame length was 8688 bits, the number of transmitted frames was 10000 . The IrCC had 17 component codes, associated with the weights $[0.049,0,0,0,0,0.24,0.16$, $0.12,0.035,0.102,0,0.071,0.093,0,0.091,0,0.039]^{1}$. We generated the FER curve for the AWGN channel model with the aid of simulation. According to the approach of [12], this will allow us to determine the average FER for arbitrary fading channels upon weighting the AWGN-FER by the PDF of the fading channel and averaging it over the legitimate dynamic range. More specifically, the channel model considered is the uncorrelated, non-dispersive Rayleigh fading channel. The average FER expression $F E R_{\text {Rayleigh }}$ is determined for the Rayleigh fading channel considered by integrating the specific $F E R_{A W G N}$ value of the AWGN channel experienced at a given SNR after weighting it by the probability of that specific SNR, which is given by:

$$
F E R_{\text {Rayleigh }}=\int_{0}^{\infty} e^{-\gamma} F E R_{A W G N}(\gamma) d \gamma,
$$

\footnotetext{
${ }^{1}$ The 17 coding coefficients $\alpha_{i}, i=[1,2, \ldots, 17]$, are the 17 coding fractions of the 17 corresponding component codes (subcode), the $i^{t h}$ of which having a code rate $\beta_{i}$ encodes the fraction $\alpha_{i}$ of the input bit stream, where we have $\left[\beta_{1}=0.1, \beta_{2}=0.15, \beta_{3}=0.2, \beta_{4}=0.25, \beta_{5}=0.3, \beta_{6}=\right.$ $0.35, \beta_{7}=0.4, \beta_{8}=0.45, \beta_{9}=0.5, \beta_{10}=0.55, \beta_{11}=0.6, \beta_{12}=$ $\left.0.65, \beta_{13}=0.7, \beta_{14}=0.75, \beta_{15}=0.8, \beta_{16}=0.85, \beta_{17}=0.9\right]$. Hence, the constraint of $R_{c}=\sum_{i=1}^{17} \alpha_{i} \beta_{i}=0.5$ is always satisfied.
} 
where $\gamma$ is the channel SNR, $e^{-\gamma}$ represents the Rayleigh channel while the $F E R_{A W G N}(\gamma)$ versus the SNR curve (obtained by off-line simulation) is approximated by the following four-segment FER vs SNR model representing the AWGN channel:

$$
F E R_{A W G N}(\gamma) \approx \begin{cases}1, & \text { if } 0 \leq \gamma<\eta_{1}, \\ 10 a_{1} \log (\gamma)+a_{2}, & \text { if } \eta_{1} \leq \gamma<\eta_{2}, \\ 10 a_{3} \log (\gamma)+a_{4}, & \text { if } \eta_{2} \leq \gamma<\eta_{3}, \\ a_{5} e^{-10 a_{6} \log (\gamma)}, & \text { if } \gamma \geq \eta_{3},\end{cases}
$$

with $\eta_{1}, \eta_{2}$ and $\eta_{3}$ being the break-points of the four-segment FER versus SNR approximation $F E R_{A W G N}(\gamma)$. Eq. (1) and Eq. (2) are suitable for approximating different FER curves by appropriately setting the corresponding parameter values invoked. Specifically, for the IrCC-URC-QPSK scheme [23] employed, we have $a_{1}=-0.5889, a_{2}=1.3341, a_{3}=$ $-3.705, a_{4}=3.5169, a_{5}=4.4669 \times 10^{6}$ and $a_{6}=18.9118$. Additionally, the values of the break-points $\eta_{1}, \eta_{2}$ and $\eta_{3}$ are determined for the SNR points of $0.6 \mathrm{~dB}, 0.7 \mathrm{~dB}$ and $0.9 \mathrm{~dB}$, whose relationships are given by:

$$
\begin{aligned}
& \eta_{1}=\frac{10^{\frac{0.6}{10}} d^{\alpha} N_{0}(4 \pi)^{2}}{P_{t_{1}} \lambda^{2}} \\
& \eta_{2}=\frac{10^{\frac{0.7}{10}} d^{\alpha} N_{0}(4 \pi)^{2}}{P_{t_{1}} \lambda^{2}} \\
& \eta_{3}=\frac{10^{\frac{0.9}{10}} d^{\alpha} N_{0}(4 \pi)^{2}}{P_{t_{1}} \lambda^{2}},
\end{aligned}
$$

where $\lambda$ is the wavelength of light, $d$ is the distance between the transmitter and the receiver, $N_{0}$ is the thermal noise power and $\alpha$ is the path-loss exponent. In this paper, we set $\alpha=2$.

Substituting our FER versus SNR model of Eq. (2) associated with the above-mentioned parameters into Eq. (1), we have the following results:

- When $0 \leq \gamma<\eta_{1}$, we have

$$
\begin{aligned}
F E R_{I} & =\int_{0}^{\eta_{1}} e^{-\gamma} d \gamma=1-e^{-\eta_{1}} \\
& =1-e^{-\frac{10 \frac{0.6}{10} d^{\alpha} N_{0}(4 \pi)^{2}}{P_{t_{1}} \lambda^{2}}}
\end{aligned}
$$

- When $\eta_{1} \leq \gamma<\eta_{2}$, we have

$$
\begin{aligned}
F E R_{I I}= & \int_{\eta_{1}}^{\eta_{2}}\left(10 a_{1} \log (\gamma)+a_{2}\right) e^{-\gamma} d \gamma \\
= & a_{2}\left(e^{-\eta_{1}}-e^{-\eta_{2}}\right) \\
& +a_{1} \int_{\eta_{1}}^{\eta_{2}} \frac{10}{\ln 10} \ln \left(\frac{P_{t_{1}} \lambda^{2}}{(4 \pi)^{2} d^{\alpha}} \gamma\right) e^{-\gamma} d \gamma ;
\end{aligned}
$$

After carrying out the integration with the aid of the Euler function of $\operatorname{Ei}(x)=\int_{-x}^{\infty} \frac{e^{-t}}{t} d t[25]$ (8.211.1), we arrive at:

$$
\begin{aligned}
F E R_{I I}= & a_{2}\left(e^{-\eta_{1}}-e^{-\eta_{2}}\right)+0.6 a_{1} e^{-\eta_{1}}-0.7 a_{1} e^{-\eta_{2}} \\
& +a_{1} \frac{10}{\ln 10}\left[E i\left(-\eta_{2}\right)-E i\left(-\eta_{1}\right)\right]
\end{aligned}
$$

- When $\eta_{2} \leq \gamma<\eta_{3}$, we have an expression similar to Eq. (8):

$$
\begin{aligned}
F E R_{I I I}= & \int_{\eta_{2}}^{\eta_{3}}\left(10 a_{3} \log (\gamma)+a_{4}\right) e^{-\gamma} d \gamma \\
= & a_{4}\left(e^{-\eta_{2}}-e^{-\eta_{3}}\right)+0.7 a_{3} e^{-\eta_{2}}-0.9 a_{3} e^{-\eta_{3}} \\
& +a_{3} \frac{10}{\ln 10}\left[E i\left(-\eta_{3}\right)-E i\left(-\eta_{2}\right)\right]
\end{aligned}
$$

- Finally, for $\gamma \geq \eta_{3}$, we have

$$
\begin{aligned}
& F E R_{I V}=\int_{\eta_{3}}^{\infty} a_{5} e^{\left(-10 a_{6} \log (\gamma)\right)} e^{-\gamma} d \gamma
\end{aligned}
$$

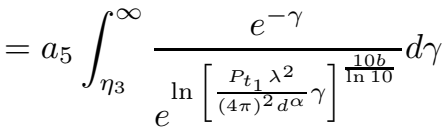

$$
\begin{aligned}
& =10^{\frac{-0.9 a_{6}}{\ln 10}} a_{5} \eta_{3} G_{1,2}^{2,0}\left[\eta_{3} \mid \begin{array}{c}
\frac{10 a_{6}}{\ln 10} \\
\frac{10 a_{6}}{\ln 10}-1,0
\end{array}\right] \text {, }
\end{aligned}
$$

where the Meijer-G function is defined in [25] (9.301) and we have $G_{1,2}^{2,0}\left[x \mid \begin{array}{c}\nu \\ \nu-1,0\end{array}\right]=E_{\nu}(x)=$ $\int_{1}^{\infty} \frac{e^{-x t}}{t^{\nu}} d t[26](06.34 .02 .001 .01)$.

However, the FER formula derived above does not consider the effects of retransmissions in the MAC layer, neither does it take into account the number of hops in the network layer. In a realistic scenario, however, we have poor channel conditions, a high level of interference, the effects of node mobility, potential network congestions and so on, where the packets are often dropped before reaching the destination. However, the dropped packets consume a high amount of energy during their passage through the network. Therefore, we analyze the Normalized Energy Consumption (NEC) during a packet's passage from the source to the destination. We have to consider two scenarios, namely the energy consumption $E_{s}$ when a packet is delivered successfully to the destination and that given by $E_{f}$ when it is dropped before reaching the destination.

The performance of a single-hop route is analyzed first, where $p_{1}$ is the successful probability of the first hop. Then, the probability $p_{s}$ that a packet is successfully delivered from the Source (S) to the destination (D) within the maximum $N_{r}$ number of retransmissions is [22]

$$
p_{s}=\sum_{i_{1}=1}^{N_{r}}\left(1-p_{1}\right)^{i_{1}-1} p_{1} .
$$

By contrast, the probability $p_{f}$ that a packet is dropped before reaching its destination is [22]

$$
p_{f}=\left(1-p_{1}\right)^{N_{r}} \text {. }
$$

Hence, the energy $E_{s}$ required for the successful transmission of a packet and that dissipated during the transmission of a failed packet, namely $E_{f}$, are respectively given by

$$
\begin{gathered}
E_{s}=\sum_{i_{1}=1}^{N_{r}}\left(1-p_{1}\right)^{i_{1}-1} p_{1} i_{1} P_{t_{1}} T, \\
E_{f}=\left(1-p_{1}\right)^{N_{r}} N_{r} P_{t_{1}} T,
\end{gathered}
$$


where $T$ is the duration of a time slot.

Consequently, the average total energy $E_{\text {total }}$ required for transmitting a packet is $E_{\text {total }}=E_{s}+E_{f}$. Furthermore, the total energy $E_{\text {total }}$ normalized by the successful probability $p_{s}$, which is the energy dissipated by the whole system during the successful delivery of a packet to $D$, can be expressed as

$$
\bar{E}_{\text {total }}=\frac{E_{\text {total }}}{p_{s}}=\frac{E_{s}+E_{f}}{p_{s}} .
$$

When substituting Eqs. (11), (13) and (14) into Eq. (15), we arrive at:

$$
\bar{E}_{\text {total }}=\frac{P_{t_{1}}}{p_{1}} T
$$

which shows that $\bar{E}_{\text {total }}$ is independent of the number of retransmissions in a single-hop route. Moreover, Eqs. (6), (7), (9) and (10) illustrate the relationship between $P_{t_{1}}$ and $p_{1}$. Then, $\bar{E}_{\text {total }}$ only depends on the distance between $\mathrm{S}$ and D. Therefore, optimizing the transmit power of the $\mathrm{S}$ may be formulated as a convex optimization problem.

Let us set the derivative of Eq. (16) with respect to $P_{t_{1}}$ to zero, yielding

$$
\begin{aligned}
\frac{1}{p_{1}}+\frac{P_{t_{1}}}{p_{1}^{2}} \frac{d\left(1-p_{1}\right)}{d P_{t_{1}}} & =0 \\
\frac{p_{1}}{-P_{t_{1}}} & =\frac{d\left(1-p_{1}\right)}{d P_{t_{1}}},
\end{aligned}
$$

Upon manipulating Eq. (17) further by setting the derivatives of the four parts of the four-segment approximated FER versus SNR curve detailed in Eqs. (6), (7), (9) and (10), we arrive at

$$
\begin{aligned}
\frac{p_{1}}{-P_{t_{1}}}= & \frac{d F E R_{1, I}}{d P_{t_{1}}}+\frac{d F E R_{1, I I}}{d P_{t_{1}}} \\
& +\frac{d F E R_{1, I I I}}{d P_{t_{1}}}+\frac{d F E R_{1, I V}}{d P_{t_{1}}} .
\end{aligned}
$$

To elaborate a little further, based on Eq. (6), we have

$$
\begin{aligned}
\frac{d F E R_{1, I}}{d P_{t_{1}}} & =\frac{d\left(1-e^{-\eta_{1}}\right)}{d P_{t_{1}}} \\
& =-\frac{10^{\frac{0.6}{10}}(4 \pi)^{2} d^{\alpha} N_{0} e^{-\frac{10 \frac{0.6}{10}(4 \pi)^{2} d^{\alpha} N_{0}}{P_{t_{1}} \lambda^{2}}}}{P_{t_{1}}^{2} \lambda^{2}} .
\end{aligned}
$$

While based on Eq. (7), we arrive at Eq. (27), where $\frac{d E i(x)}{d x}=\frac{e^{x}}{x}$ [25] (8.211.1). It can be readily shown that $\frac{d F E R_{1, I I I}}{d P_{t}}$ obeys an expression similar to Eq. (27), which is formulated in Eq. (28).

Finally, based on Eq. (10), the 4th part at the righthand side of Eq. (18) can be expressed as Eq. (29), where the differentiation of Meijer's G function was taken from [26] (07.34.20.0005.01). Eqs. (27), (28) and (29) are shown on the top of next page.

Finally, when substituting Eqs. (19), (27), (28) and (29) into Eq. (18), the optimized transmit power $P_{t_{1}}$ can be found.

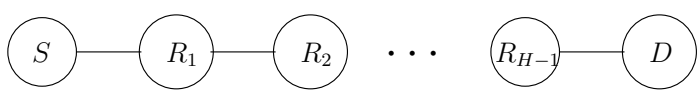

Fig. 1. Test-topology having one source, one destination and $(H-1)$ relay nodes.

\section{B. Theoretical analysis of TR in an idealized network}

The energy consumption of an idealized multi-hop route is analyzed in this subsection. The network topology is shown in Fig. 1.

In Fig. 1 , we have a single $S$, a single $D$ and $(H-1)$ $R$ s. The $(H-1) R$ s are located between $S$ and $D$. Our previous contribution [22] analyzed both the probability and the total energy consumption of a packet, when it is delivered successfully to $D$ or when it is dropped before reaching $D$ of Fig. 1. However, in [22] we assumed that the transmit power of all nodes is the same, which wasted some energy in the realistic scenario, when the distances between each pair of nodes was different. If the optimal distance-dependent transmit power is found, then the NEC may be further reduced.

The probabilities $p_{s}$ and $p_{f}$ are the same as those in [22], namely

$$
\begin{aligned}
p_{s}= & \sum_{i_{1}=1}^{N_{r}} \cdots \sum_{i_{H}=1}^{N_{r}}\left(1-p_{1}\right)^{i_{1}-1} p_{1}\left(1-p_{2}\right)^{i_{2}-1} p_{2} \\
& \cdots\left(1-p_{H}\right)^{i_{H}-1} p_{H}, \\
p_{f}= & p_{f}(1)+\sum_{h=2}^{H} p_{f}(h),
\end{aligned}
$$

where $p_{f}(1)$ is given by Eq. (12) and $p_{f}(h)$ is the probability that a packet is dropped at the $h$-th hop, which is formulated as:

$$
\begin{aligned}
p_{f}(h)= & \sum_{i_{1}=1}^{N_{r}} \cdots \sum_{i_{h-1}=1}^{N_{r}}\left(1-p_{1}\right)^{i_{1}-1} p_{1} \ldots \\
& \left(1-p_{h-1}\right)^{i_{h-1}-1} p_{h-1}\left(1-p_{h}\right)^{N_{r}}, h \neq 1 .
\end{aligned}
$$

Let $E_{i}$ be the energy required by node $i$ to send a packet, where $E_{i}=P_{t_{i}} T$. Then, we can show that $E_{s}$ and $E_{f}$ are formulated as:

$$
\begin{aligned}
E_{s}= & \sum_{i_{1}=1}^{N_{r}} \cdots \sum_{i_{H}=1}^{N_{r}}\left(1-p_{1}\right)^{i_{1}-1} p_{1}\left(1-p_{2}\right)^{i_{2}-1} p_{2} \\
& \cdots\left(1-p_{H}\right)^{i_{H}-1} p_{H}\left(i_{1} E_{1}+i_{2} E_{2}+\cdots+i_{H} E_{H}\right),
\end{aligned}
$$

$$
\begin{aligned}
E_{f}= & E_{f}(1)+\sum_{h=2}^{H} E_{f}(h) \\
= & N_{r} E_{1}\left(1-p_{1}\right)^{N_{r}}+\sum_{h=2}^{H}\left[\sum_{i_{1}=1}^{N_{r}} \cdots \sum_{i_{h-1}=1}^{N_{r}}\left(1-p_{1}\right)^{i_{1}-1} p_{1}\right. \\
& \cdots\left(1-p_{h-1}\right)^{i_{h-1}-1} p_{h-1}\left(1-p_{h}\right)^{N_{r}}\left(i_{1} E_{1}\right. \\
& \left.\left.+\cdots+i_{h-1} E_{h-1}+N_{r} E_{h}\right)\right] .
\end{aligned}
$$

Let $D_{s}$ denote the average time required for delivering a packet successfully to $D$, where the source of delay is assumed to be the Automatic Repeat reQuest (ARQ)-aided 
retransmissions. Explicitly, each new hop and new transmission attempt imposes a delay of unity, i.e. one time-slot (TS). Furthermore, let $D_{f}$ be the average delay imposed on a packet's passage through the route, when it is dropped before reaching its destination. Hence, the total delay is represented as $D_{\text {total }}=D_{s}+D_{f}$. These average delays have been quantified in [22], which are

$$
\begin{gathered}
D_{s}=\left[\sum_{i_{1}=1}^{N_{r}} \cdots \sum_{i_{H}=1}^{N_{r}}\left(1-p_{1}\right)^{i_{1}-1} p_{1}\left(1-p_{2}\right)^{i_{2}-1} p_{2}\right. \\
\left.\cdots\left(1-p_{H}\right)^{i_{H}-1} p_{H}\left(i_{1}+i_{2}+\cdots+i_{H}\right)\right] T, \\
D_{f}=p_{f}(1) N_{r} T+\sum_{h=2}^{H} D_{f}(h),
\end{gathered}
$$

where $D_{f}(h)$ is the average delay experienced by a packet, which is dropped during the $h$-th hop, expressed as

$$
\begin{aligned}
D_{f}(h)= & {\left[\sum_{i_{1}=1}^{N_{r}} \cdots \sum_{i_{h-1}=1}^{N_{r}}\left(1-p_{1}\right)^{i_{1}-1} p_{1} \cdots\right.} \\
& \left(1-p_{h-1}\right)^{i_{h-1}-1} p_{h-1}\left(1-p_{h}\right)^{N_{r}} \\
& \left.\left(i_{1}+\cdots+i_{h-1}+N_{r}\right)\right] T, h \neq 1 .
\end{aligned}
$$

For the sake of simplifying Eq. (23), (24), (25) and (26), we define $A\left(p_{i}\right)=\left(\frac{1-\left(1-p_{i}\right)^{N_{r}}}{p_{i}}-N_{r}\left(1-p_{i}\right)^{N_{r}}\right) E_{i}$ and $B\left(p_{i}\right)=1-\left(1-p_{i}\right)^{N_{r}}$. Then, we have $p_{s}=\prod_{1}^{H} B\left(p_{i}\right)$. Furthermore, it may be readily shown that $E_{s}$ and $E_{f}$ can be formulated alternatively as:

$$
\begin{gathered}
E_{s}=\left[\prod_{i=1}^{H} B\left(p_{i}\right)\right]\left[\sum_{i=1}^{H} \frac{A\left(p_{i}\right)}{B\left(p_{i}\right)}\right], \\
E_{f}=\sum_{h=2}^{H}\left[\left[\prod_{i=1}^{h-1} B\left(p_{i}\right)\right]\left[\sum_{i=1}^{h-1} \frac{A\left(p_{i}\right)}{B\left(p_{i}\right)}\right]\left[1-B\left(p_{h}\right)\right]\right. \\
\left.+N_{r} E_{h}\left(1-B\left(p_{h}\right)\right) \prod_{i=1}^{h-1} B\left(p_{i}\right)\right]+N_{r} E_{1}\left[1-B\left(p_{1}\right)\right] .
\end{gathered}
$$

Similarly, upon defining $C\left(p_{i}\right)=\left(\frac{1-\left(1-p_{i}\right)^{N_{r}}}{p_{i}}-N_{r}(1-\right.$ $\left.\left.p_{i}\right)^{N_{r}}\right) T$, we can express $D_{s}$ and $D_{f}$ as

$$
\begin{gathered}
D_{s}=\left[\prod_{i=1}^{H} B\left(p_{i}\right)\right]\left[\sum_{i=1}^{H} \frac{C\left(p_{i}\right)}{B\left(p_{i}\right)}\right] \\
D_{f}=\sum_{h=2}^{H}\left[\left[\prod_{i=1}^{h-1} B\left(p_{i}\right)\right]\left[\sum_{i=1}^{h-1} \frac{C\left(p_{i}\right)}{B\left(p_{i}\right)}\right]\left[1-B\left(p_{h}\right)\right]\right. \\
\left.+N_{r} T\left[1-B\left(p_{h}\right)\right] \prod_{i=1}^{h-1} B\left(p_{i}\right)\right]+N_{r} T\left[1-B\left(p_{1}\right)\right] .
\end{gathered}
$$

Based on the above derivation, the NEC expressed as $\bar{E}_{\text {total }}=\frac{E_{\text {total }}}{p_{s}}=\frac{E_{s}+E_{f}}{p_{s}}$ can now be evaluated.

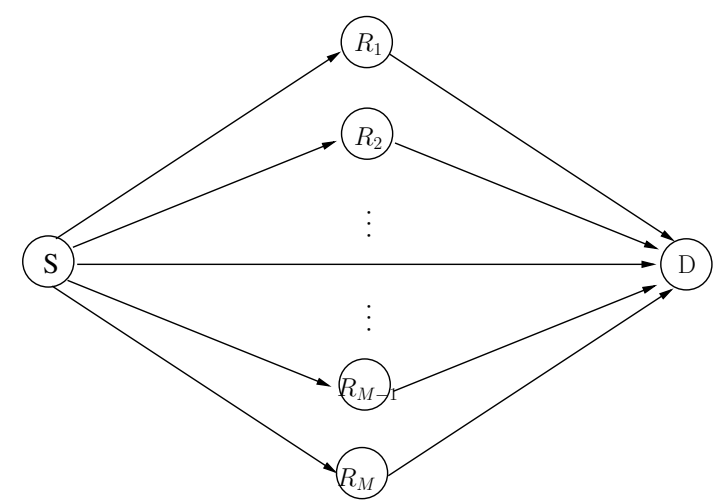

Fig. 2. A two-hop network assisted by a number of relays.

The end-to-end delay $D_{e 2 e}$ is given by

$$
D_{e 2 e}=D_{s},
$$

which represents the delay experienced by a packet that is successfully delivered to the destination. Moreover, the endto-end throughput $R_{e 2 e}$ is given by

$$
R_{e 2 e}=\frac{p_{s}}{D_{\text {total }}}=\frac{p_{s}}{D_{s}+D_{f}} .
$$

\section{Theoretical analysis of $O R$ in a random network}

The TR transmits the packet along the specific pre-selected route having the lowest estimated NEC. This pre-selected route is determined after the estimation and comparison of the NEC of each potential candidate route. The information invoked for routing decisions is gleaned during the process of route discovery, but this information may become stale owing to node-mobility. Instead, OR considers the potential chances of success for each candidate relay, bearing in mind their timevariant channel conditions. Regardless of which particular relay receives the packet from the source successfully, if this relay has the highest priority in the forwarder relay list, it will forward the packet to the next relay. Naturally, the challenge in the design of the OR procedure is the beneficial selection of the forwarder R-set, the specific priority order of the potential forwarders and the avoidance of duplicate transmissions [16]. We assume that all the nodes in a node's neighbor list belong to this node's forwarder R-list. The metric used for determining the priority order is the normalized energy required by this particular relay for reaching D. Acknowledgement (ACK) packets are employed for avoiding the duplicate transmissions. The particular relay in the forwarder R-set, which has the highest priority owing to requiring the lowest energy will send the ACK first. The other relays, which overhear the ACK will withdraw from the competition [27,28].

A two-hop network is shown in Fig. 2, which has a single source $S$, a single destination $D$ and $M$ relays $R_{1}, R_{2}, \cdots, R_{M-1}, R_{M} . S$ and $D$ are capable of communicating with all the relays, as well as with each other. By contrast, the $M$ relays are unable to communicate with each other. We stipulate the idealized simplifying assumption furthermore that each node knows the position of all other nodes. For each relay $R_{m}, m=1 \ldots M$, the total average energy consumption $E_{R_{m} D}$ required for transmission from $R_{m}$ to $D$ is given by $E_{R_{m} D}=E_{R_{m} D}^{s}+E_{R_{m} D}^{f}$, where 


$$
\begin{aligned}
& \frac{d F E R_{1, I I}}{d P_{t_{1}}}=\frac{d\left[a_{2}\left(e^{-\eta_{1}}-e^{-\eta_{2}}\right)\right]}{d P_{t_{1}}}+\frac{d\left[0.6 a_{1} e^{-\eta_{1}}-0.7 a_{1} e^{-\eta_{2}}+a_{1} \frac{10}{\ln 10}\left[E i\left(-\eta_{2}\right)-E i\left(-\eta_{1}\right)\right]\right]}{d P_{t_{1}}} \\
& =-\left(0.6 a_{1}+a_{2}\right) \frac{10^{\frac{0.6}{10}}(4 \pi)^{2} d^{\alpha} N_{0} e^{-\frac{10 \frac{0.6}{10}(4 \pi)^{2} d^{\alpha} N_{0}}{P_{t_{1}} \lambda^{2}}}}{P_{t_{1}}^{2} \lambda^{2}}+\left(a_{2}+0.7 a_{1}\right) \frac{10^{\frac{0.7}{10}}(4 \pi)^{2} d^{\alpha} N_{0} e^{-\frac{10 \frac{0.7}{10}(4 \pi)^{2} d^{\alpha} N_{0}}{P_{t_{1} \lambda^{2}}}}}{P_{t_{1}}^{2} \lambda^{2}} \\
& +\frac{10 a_{1}}{P_{t_{1}} \ln 10}\left[e^{-\frac{10 \frac{0.6}{10}(4 \pi)^{2} d^{\alpha} N_{0}}{P_{t_{1}} \lambda^{2}}}-e^{-\frac{10 \frac{0.7}{10}(4 \pi)^{2} d^{\alpha} N_{0}}{P_{t_{1}} \lambda^{2}}}\right] \\
& \frac{d F E R_{1, I I I}}{d P_{t_{1}}}=\frac{d\left[a_{4}\left(e^{-\eta_{2}}-e^{-\eta_{3}}\right)\right]}{d P_{t_{1}}}+\frac{d\left[0.7 a_{3} e^{-\eta_{2}}-0.9 a_{3} e^{-\eta_{3}}+a_{3} \frac{10}{\ln 10}\left[E i\left(-\eta_{3}\right)-E i\left(-\eta_{2}\right)\right]\right]}{d P_{t_{1}}} \\
& =-\left(0.7 a_{3}+a_{4}\right) \frac{10^{\frac{0.7}{10}}(4 \pi)^{2} d^{\alpha} N_{0} e^{-\frac{10 \frac{0.7}{10}(4 \pi)^{2} d^{\alpha} N_{0}}{P_{t_{1}} \lambda^{2}}}}{P_{t_{1}}^{2} \lambda^{2}}+\left(a_{4}+0.9 a_{3}\right) \frac{10^{\frac{0.9}{10}}(4 \pi)^{2} d^{\alpha} N_{0} e^{-\frac{10 \frac{0.9}{10}(4 \pi)^{2} d^{\alpha} N_{0}}{P_{t_{1}} \lambda^{2}}}}{P_{t_{1}}^{2} \lambda^{2}} \\
& +\frac{10 a_{3}}{P_{t_{1}} \ln 10}\left[e^{-\frac{10 \frac{0.7}{10}(4 \pi)^{2} d^{\alpha} N_{0}}{P_{t_{1}} \lambda^{2}}}-e^{-\frac{10 \frac{0.9}{10}(4 \pi)^{2} d^{\alpha} N_{0}}{P_{t_{1}} \lambda^{2}}}\right] \\
& \frac{d F E R_{1, I V}}{d P_{t_{1}}}=\frac{d\left[10^{\frac{-0.9 a_{6}}{\ln 10}} a_{5} \eta_{3} E_{\frac{10 a_{6}}{\ln 10}}\left(\eta_{3}\right)\right]}{d P_{t_{1}}} \\
& =-10^{\frac{-0.9 a_{6}}{10}} a_{5} G_{2,3}^{2,1}\left[\frac{10^{\frac{0.9}{10}}(4 \pi)^{2} d^{\alpha} N_{0}}{P_{t_{1}} \lambda^{2}} \mid \begin{array}{c}
-1, \frac{10 a_{6}}{\ln 10} \\
\frac{10 a_{6}}{\ln 10}-1,0,0
\end{array}\right] \frac{10^{\frac{0.9}{10}}(4 \pi)^{2} d^{\alpha} N_{0}}{P_{t_{1}}^{2} \lambda^{2}} .
\end{aligned}
$$

$E_{R_{m} D}^{s}$ and $E_{R_{m} D}^{f}$ are given in Eq. (13) and (14). Let $E_{S}$ denote the energy dissipated while sending a packet from the source $S$ to any of the relays $R_{m}$, which is $E_{S}=P_{t_{S}} T$. We assume that $E_{R_{1} D}<E_{R_{2} D}<\cdots<E_{R_{M} D}$. Furthermore, for convenience, we represent the destination node $D$ as $R_{0}$ and define $\prod_{m=0}^{M}\left(1-p_{S R_{m}}\right)=\zeta$, where $p_{S R_{m}}$ is the probability of a packet, which is successfully delivered from $S$ to $R_{m}$.

If the source successfully sends a packet to the $m$-th relay, $m=0,1, \ldots, M$, with the aid of $n_{r}$ transmissions, the probability of this event is

$$
\begin{aligned}
& p_{0}\left(n_{r}\right)=\zeta^{n_{r}-1} p_{S R_{0}}, \text { if } m=0 \\
& p_{m}\left(n_{r}\right)=\zeta^{n_{r}-1} \prod_{i=0}^{m-1}\left(1-p_{S R_{i}}\right) p_{S R_{m}}, \text { if } 1 \leq m \leq M
\end{aligned}
$$

Correspondingly, the energy dissipated becomes

$$
\begin{aligned}
E_{0}\left(n_{r}\right) & =n_{r} E_{S}, \text { if } m=0 \\
E_{m}\left(n_{r}\right) & =n_{r} E_{S}+E_{R_{m} D}, \text { if } 1 \leq m \leq M .
\end{aligned}
$$

Let $D_{R_{m} D}$ denote the average delay of a packet traversing from $R_{m}, m=1, \ldots, M$, to $D$, including the delay $D_{R_{m} D}^{s}$ encountered by a packet that is successfully delivered to $D$ and the delay $D_{R_{m} D}^{f}$ experienced when a packet is dropped before reaching $D$. We then have $D_{R_{m} D}=D_{R_{m} D}^{s}+D_{R_{m} D}^{f}$, where $D_{R_{m} D}^{s}$ represents $D_{s}$ expressed by Eq. (33) and $D_{R_{m} D}^{f^{\prime}}$ corresponds to $D_{f}$ expressed by Eq. (34), provided that the number of hops is 1 . Consequently, we have:

$$
\begin{aligned}
D_{0}\left(n_{r}\right) & =n_{r} D_{S}, \text { if } m=0 \\
D_{m}\left(n_{r}\right) & =n_{r} D_{S}+D_{R_{m} D} \\
& =n_{r} D_{S}+\left(D_{R_{m} D}^{s}+D_{R_{m} D}^{f}\right), \text { if } 1 \leq m \leq M
\end{aligned}
$$

where $D_{S}$ is $T$, which denotes the duration of a TS.

Consequently, when taking into account all the possible events, the total energy consumption is

$$
\begin{aligned}
E_{\text {total }}= & \sum_{n_{r}=1}^{N_{r}} p_{0}\left(n_{r}\right) E_{0}\left(n_{r}\right) \\
& +\sum_{n_{r}=1}^{N_{r}} \sum_{m=1}^{M} p_{m}\left(n_{r}\right) E_{m}\left(n_{r}\right)+\zeta^{N_{r}}\left(N_{r} E_{S}\right),
\end{aligned}
$$

while the total delay becomes:

$$
\begin{aligned}
D_{\text {total }}= & \sum_{n_{r}=1}^{N_{r}} p_{0}\left(n_{r}\right) D_{0}\left(n_{r}\right) \\
& +\sum_{n_{r}=1}^{N_{r}} \sum_{m=1}^{M} p_{m}\left(n_{r}\right) D_{m}\left(n_{r}\right)+\zeta^{N_{r}}\left(N_{r} D_{S}\right),
\end{aligned}
$$

where the final term in Eq. (43) and Eq. (44) is contributed by the event that the packet was not delivered by the source $S$ even after $N_{r}$ attempts. Upon substituting Eq. (38) and 
Eq. (40) into Eq. (43), we obtain

$$
\begin{aligned}
E_{\text {total }}= & \sum_{n_{r}=1}^{N_{r}} \zeta^{n_{r}-1} p_{S R_{0}}\left(n_{r} E_{S}\right) \\
& +\sum_{n_{r}=1}^{N_{r}} \sum_{m=1}^{M} \zeta^{n_{r}-1} \prod_{i=0}^{m-1}\left(1-p_{S R_{i}}\right) p_{S R_{m}}\left(n_{r} E_{S}\right. \\
& \left.+E_{R_{m} D}\right)+\zeta^{N_{r}}\left(N_{r} E_{S}\right) .
\end{aligned}
$$

After substituting Eq. (38) and Eq. (42) into Eq. (44), we arrive at Eq. (46), where the second term is contributed by the event that a packet is successfully delivered from the source $S$ to the destination $D$, while the third term by the event that a packet is successfully delivered from the source $S$ to the relay $R_{m}, m=1, \ldots, M$, but it is dropped during its passage from the relay $R_{m}, m=1, \ldots, M$, to the destination $D$.

After further simplifications, Eq. (43) and Eq. (44) may be written as Eq. (47) and Eq. (48). Then, the end-to-end delay $D_{e 2 e}$ is given by the first two terms of Eq. (48), formulated as Eq. (49). Eqs. (46), (47), (48) and Eq. (49) are shown on the top of next page.

Meanwhile, the packet transmitted from $S$ may be dropped in the $S D, S-R_{m}$ or $R_{m}-D$ link, where we have $m=$ $1, \ldots, M$ and again, the destination can be replaced by $R_{0}$. The probabilities of these events are given by

$$
\begin{aligned}
p_{f, S-R_{m}}= & \zeta^{N_{r}}, m=0, \ldots, M \\
p_{f, R_{m}-D}= & \sum_{n_{r}=1}^{N_{r}} \sum_{m=1}^{M} \zeta^{n_{r}-1}\left[\prod_{j=0}^{m-1}\left(1-p_{S R_{j}}\right)\right] \\
& p_{S R_{m}}\left(1-p_{R_{m} D}\right), m=1, \ldots, M,
\end{aligned}
$$

where $p_{R_{m} D}$ is the probability that a packet is successfully delivered from $R_{m}, m=1, \ldots, M$, to the destination $D$.

Then we may formulate the end-to-end outage probability $p_{f}$ as

$$
p_{f}=p_{f, S-R_{0}}+p_{f, S-R_{m}}+p_{f, R_{m}-D}, m=1, \ldots, M \text {. }
$$

Upon substituting Eq. (51) into Eq. (52), we arrive at:

$$
\begin{gathered}
p_{f}=\zeta^{N_{r}}+\sum_{n_{r}=1}^{N_{r}} \sum_{m=1}^{M} \zeta^{n_{r}-1}\left[\prod_{j=0}^{m-1}\left(1-p_{S R_{j}}\right)\right] \\
P_{S R_{m}}\left(1-p_{R_{m} D}\right) \\
=\zeta^{N_{r}}+\frac{1-\zeta^{N_{r}}}{1-\zeta} \sum_{m=1}^{M}\left[\prod_{j=0}^{m-1}\left(1-p_{S R_{j}}\right)\right] \\
P_{S R_{m}}\left(1-p_{R_{m} D}\right) .
\end{gathered}
$$

Furthermore, we may formulate the NEC $\bar{E}_{\text {total }}$ as

$$
\bar{E}_{t o t a l}=\frac{E_{t o t a l}}{1-p_{f}}
$$

while the end-to-end throughput $R_{e 2 e}$ is given by

$$
R_{e 2 e}=\frac{p_{s}}{D_{\text {total }}}=\frac{1-p_{f}}{D_{\text {total }}}
$$

Let us now introduce a new low-complexity algorithm in the next section for calculating both the delay $D_{e 2 e}$ and the throughput $R_{e 2 e}$ in a simpler way. Moreover, we will demonstrate that our analysis of the energy consumption can be extended to a large network synthesized by numerous twohop networks, as shown in Fig. 2. The corresponding details are provided in Section III-A.

\section{Routing Algorithms}

\section{A. TR algorithm}

The performance of the single-hop route and of an idealized network was characterized in Subsections II-A and II-B, which may be readily extended to existing routing protocols, such as the DSR (Dynamic Source Routing), AODV (Ad hoc On-demand Distance Vector) and DYMO (DYnamic Manet On-demand) routing protocols. During the route discovery process, the routing packets are used for gathering the necessary information and for feeding it back to the source. Then the source makes the final decision required for sending the data packets. The most important feature of traditional routing is that the route is selected first, then the packets are always delivered along this particular route, until it is broken, for example due to node-mobility. At that moment, a sub-optimal candidate route is chosen by route-repair, or the route rediscovery process will be re-activated for finding a totally new route.

The idealized multi-hop network of Fig. 1 may be extended to a more realistic random network relying on Dijkstra's routing algorithm [29] and invoking the NEC $\bar{E}_{\text {total }}$ for route selection. The routing algorithm is described in Algorithm 1. We assume that $\mathcal{V}$ is the vertex set, $v$ is a node in the set $\mathcal{V}, \mathcal{F}(v)$ denotes the predecessor set of nodes in the route before the node $v$, while $\bar{E}(v), \bar{E}(S, v)$ denote the NEC and $p_{s}(v), p_{s}(S, v)$ represent the successful probability of a packet from the source $S$ to the node $v$. Furthermore, $D_{s}(v)$ and $D_{s}(S, v)$ denote the delay encountered by a packet delivered successfully from the source $S$ to the node $v$, while $D_{f}(v)$ and $D_{f}(S, v)$ represent the delay of a packet that was dropped before reaching the node $v$. Specifically, $\bar{E}(S, v), p_{s}(S, v)$, $D_{s}(S, v)$ and $D_{f}(S, v)$ are the intermediate values of $\bar{E}(v)$, $p_{s}(v), D_{s}(v)$ and $D_{f}(v)$, respectively. Moreover, $\mathcal{S}$ represents the set of selected nodes, while $P_{t}^{\text {opt }}(u, v)$ denotes the optimal transmit power of node $u$ assigned for transmission to node $v$.

Lines 1-3 initialize the predecessor set $\mathcal{F}(v)$ of each node and the NEC between $S$ and each other node. In line 4, the NEC, the delay and the successful delivery probability are set to zero and $S$ is selected for inclusion into $\mathcal{S}$. In line 8, the notation $u$ represents the specific node, which has just been incorporated into $\mathcal{S}$ in the previous loop. Initially, only $S$ belongs to $\mathcal{S}$. Therefore, $S$ is $u$. $S$ has a neighbor node set. For each node $v$, which belongs to the neighbor set of $S$, we can calculate the NEC $\bar{E}(S, v)$ between $S$ and $v$, given that $S$ employs the optimal transmit power. The optimal transmit power $P_{t}^{\text {opt }}(u, v)$ of node $u$ in the link ' $u-v$ ' is found by identifying the lowest NEC between $S$ and $v$, which is obtained by setting the derivative of Eq. (15) with respect to $P_{t_{u}}$ to zero. Meanwhile, lines 9-11 show that if the NEC $\bar{E}(S, v)$ from $S$ to $v$ via $u$ becomes lower than the stored $\bar{E}(v)$, then the NEC $\bar{E}(v)$ and the corresponding delay from $S$ to $v$ are updated. In lines $12-14$, the specific node, which 


$$
\begin{aligned}
& D_{\text {total }}=\sum_{n_{r}=1}^{N_{r}} \zeta^{n_{r}-1} p_{S R_{0}}\left(n_{r} D_{S}\right)+\sum_{n_{r}=1}^{N_{r}} \sum_{m=1}^{M} \zeta^{n_{r}-1} \prod_{i=0}^{m-1}\left(1-p_{S R_{i}}\right) p_{S R_{m}}\left(n_{r} D_{S}+D_{R_{m} D}^{s}+D_{R_{m} D}^{f}\right)+\zeta^{N_{r}}\left(N_{r} D_{S}\right) \\
& =\sum_{n_{r}=1}^{N_{r}} \zeta^{n_{r}-1} p_{S R_{0}}\left(n_{r} D_{S}\right)+\sum_{n_{r}=1}^{N_{r}} \sum_{m=1}^{M} \zeta^{n_{r}-1} \prod_{i=0}^{m-1}\left(1-p_{S R_{i}}\right) p_{S R_{m}}\left(n_{r} D_{S}+D_{R_{m} D}^{s}\right) \\
& +\sum_{n_{r}=1}^{N_{r}} \sum_{m=1}^{M} \zeta^{n_{r}-1} \prod_{i=0}^{m-1}\left(1-p_{S R_{i}}\right) p_{S R_{m}}\left(n_{r} D_{S}+D_{R_{m} D}^{f}\right)+\zeta^{N_{r}}\left(N_{r} D_{S}\right) \\
& E_{\text {total }}=E_{S}\left(\frac{1-\zeta^{N_{r}}}{1-\zeta}-N_{r} \zeta^{N_{r}}\right)+\frac{1-\zeta^{N_{r}}}{1-\zeta} \sum_{m=1}^{M}\left[E_{R_{m} D} p_{S R_{m}}\left[\prod_{j=0}^{m-1}\left(1-p_{S R_{j}}\right)\right]\right]+N_{r} E_{S} \zeta^{N_{r}} \\
& D_{\text {total }}=D_{S}\left(\frac{1-\zeta^{N_{r}}}{1-\zeta}-N_{r} \zeta^{N_{r}}\right)+\frac{1-\zeta^{N_{r}}}{1-\zeta} \sum_{m=1}^{M}\left[D_{R_{m} D}^{s} p_{S R_{m}}\left[\prod_{j=0}^{m-1}\left(1-p_{S R_{j}}\right)\right]\right] \\
& +\frac{1-\zeta^{N_{r}}}{1-\zeta} \sum_{m=1}^{M}\left[D_{R_{m} D}^{f} p_{S R_{m}}\left[\prod_{j=0}^{m-1}\left(1-p_{S R_{j}}\right)\right]\right]+N_{r} D_{S} \zeta^{N_{r}} \\
& D_{e 2 e}=D_{s}=D_{S}\left(\frac{1-\zeta^{N_{r}}}{1-\zeta}-N_{r} \zeta^{N_{r}}\right)+\frac{1-\zeta^{N_{r}}}{1-\zeta} \sum_{m=1}^{M}\left[D_{R_{m} D}^{s} p_{S R_{m}}\left[\prod_{j=0}^{m-1}\left(1-p_{S R_{j}}\right)\right]\right] .
\end{aligned}
$$

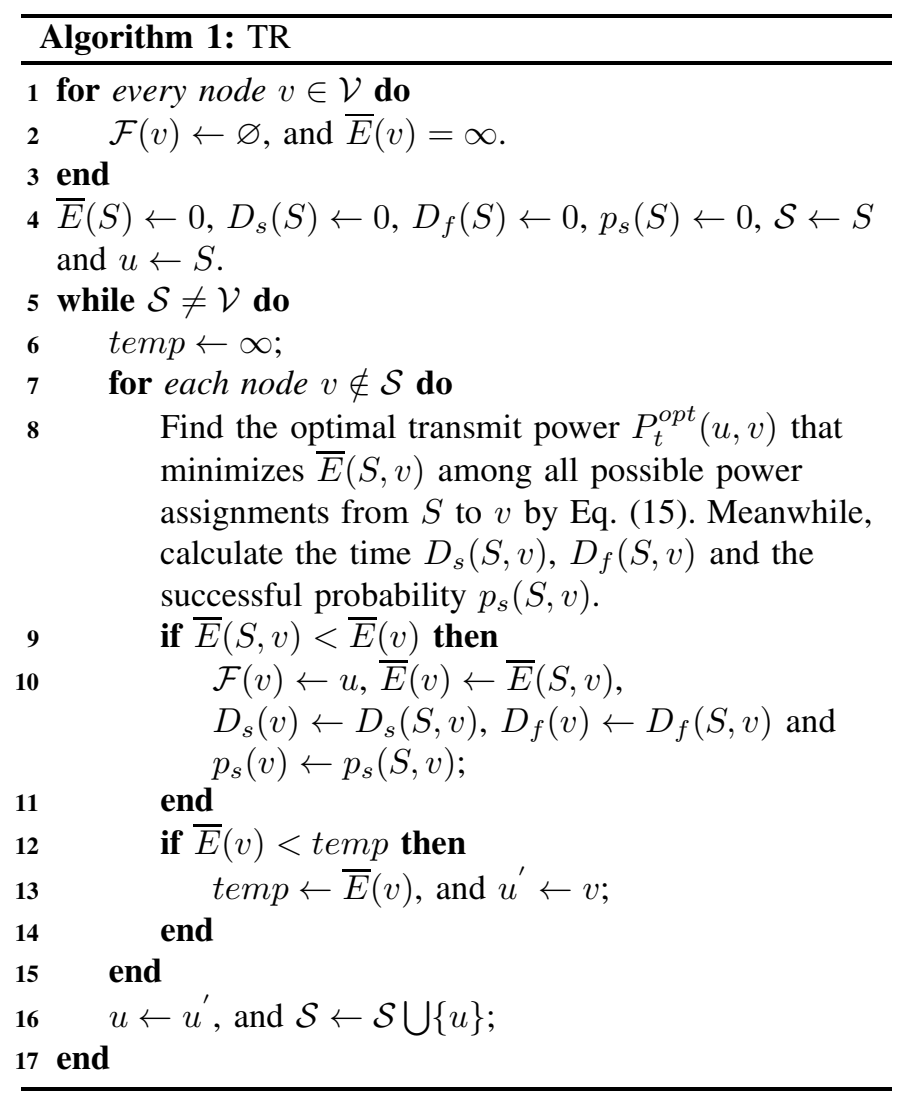

has the lowest $\bar{E}_{S, v}$ amongst all $u$ 's neighbor set, would be incorporated into $\mathcal{S}$ and becomes the new $u$ in the next loop, as seen in line 16. Again, $u$ has a new neighbor set and the next new $u$ will be selected from the neighbor set. When we have $\mathcal{S}=\mathcal{V}$, Algorithm 1 converges, as shown in line 5 . Moreover, this algorithm is a locally - rather than globally optimal algorithm, which is in fact optimal for every single hop. This is justified by the calculation of the optimal transmit power $P_{t}^{o p t}(u, v)$ in line 8 . Therefore, the source $S$ employs Algorithm 1 for identifying that particular route, which has the lowest NEC $\bar{E}_{\text {total }}$. At the same time, it also determines the delay $D_{s}(S)$, namely, the end-to-end delay $D_{e 2 e}$ of the selected route. Finally, the end-to-end throughput $R_{e 2 e}$ may be calculated by Eq. (36) based on $D_{s}(S), D_{f}(S)$ and $p_{s}(S)$.

Fig. 3 shows the step-by-step execution of Algorithm 1 using the NEC metric $\bar{E}_{\text {total }}$. The positions of $S, D, R_{1}$ and $R_{2}$ are $(100,100),(900,100),(500,500)$ and $(300,400)$, respectively. The other relevant parameters are listed in Table I. We assume that each node is aware of the other nodes' position, hence also of their distance. In a compact form, we have $\mathcal{V}=\left\{S, R_{1}, R_{2}, D\right\}$ and $\mathcal{S}=\{S\}$, as shown in Fig. 3(a). In Fig. 3(b), $S$ calculates its transmit power optimized for minimizing the NEC from Eq. (15), which is $\bar{E}_{S R_{1}}=1.8 \times 10^{-8} \mathrm{~mJ} / \mathrm{bit}, \bar{E}_{S R_{2}}=0.7 \times 10^{-8} \mathrm{~mJ} / \mathrm{bit}$, $\bar{E}_{S D}=3.5 \times 10^{-8} \mathrm{~mJ} /$ bit for transmission from $S$ to $R_{1}$, $R_{2}$ and $D$, respectively, as also seen in line 8 of Algorithm 1. Since $\bar{E}_{S R_{2}}=0.7 \times 10^{-8} \mathrm{~mJ} / \mathrm{bit}$ is the lowest in the set of the three energies, we update $\mathcal{S}$ to $\left\{S, R_{2}\right\}$, as shown in line 13 and line 16 of Algorithm 1. Then in Fig. 3(c), $R_{2}$ 


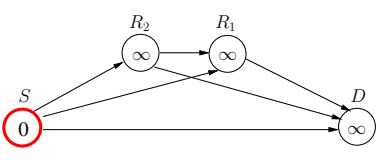

(a)

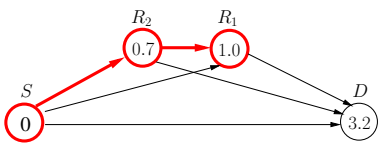

(c)

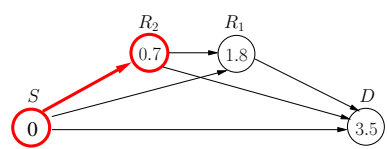

(b)

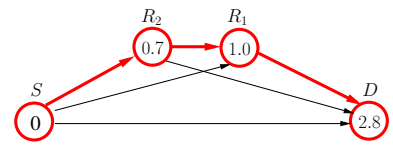

(d)
Fig. 3. Execution of Algorithm 1. The value within a node $v$ is its energy cost $\bar{E}_{\text {total }}\left(\times 10^{-8} \mathrm{~mJ} / \mathrm{bit}\right)$ for transmission from $S$ to node $v$. After each iteration one node is incorporated into the set $\mathcal{S}$. The nodes in boldface denote the nodes in $\mathcal{S}$ after each iteration and the arrows in boldface represent the shortest route from $S$ to the nodes in boldface after each iteration. Due to the adjustable transmit power of node $u$, the probability $p_{f}(u, v)$ of a packet, which is dropped at any link $u-v$ after $N_{r}=7$ retransmissions, has nearly the same value of $p_{f}(u, v)=0.041$, hence we do not plot this value next to the arrows for simplicity. (a) The situation just after the initialization, where $\mathcal{S}=\{S\}$. (b) The first iteration of the algorithm, where we have $\mathcal{S}=\{S\}$ before the iteration, while after the iteration $R_{2}$ is incorporated into the set $\mathcal{S}$ with the optimum power of $P_{t}^{o p t}\left(S, R_{2}\right)=0.16 \mathrm{~mW}$, yielding $\mathcal{S}=\left\{S, R_{2}\right\}$. (c) The second iteration of the algorithm, where we have $\mathcal{S}=\left\{S, R_{2}\right\}$ before the iteration, while after the iteration $R_{1}$ is incorporated into the set $\mathcal{S}$ with the optimum power of $P_{t}^{o p t}\left(R_{2}, R_{1}\right)=0.06 \mathrm{~mW}$, yielding $\mathcal{S}=\left\{S, R_{2}, R_{1}\right\}$. (d) The final iteration of the algorithm, where we have $\mathcal{S}=\left\{S, R_{2}, R_{1}\right\}$ before the iteration, while after the iteration $D$ is incorporated into the set $\mathcal{S}$ with the optimum power of $P_{t}^{\text {opt }}\left(R_{1}, D\right)=0.39 \mathrm{~mW}$, yielding $\mathcal{S}=\left\{S, R_{2}, R_{1}, D\right\}$. The algorithm terminates.

calculates its transmit power optimized for minimizing the NEC from Eq. (15) for the transmission, which is spanning from $S$ to node $R_{1}$ and $D$ via $R_{2}$, respectively, as shown in line 9 of Algorithm 1. Since the updated NEC $\bar{E}_{S R_{1}}=$ $1.0 \times 10^{-8} \mathrm{~mJ} / \mathrm{bit}$ is lower than $\bar{E}_{S D}=3.2 \times 10^{-8} \mathrm{~mJ} / \mathrm{bit}$, $\mathcal{S}$ is updated to $\left\{S, R_{2}, R_{1}\right\}$, as shown in line 13 and line 16 of Algorithm 1. Finally, in Fig. 3(d), $R_{1}$ adjusts its own transmit power to the optimal one, which minimizes the NEC $\bar{E}_{S D}=2.8 \times 10^{-8} \mathrm{~mJ} / \mathrm{bit}$ from $S$ to $D$ via $R_{2}$ and $R_{1}$, as shown in line 8 of Algorithm 1. At this stage, $D$ is incorporated into $\mathcal{S}$. Since we have $\mathcal{S}=\left\{S, R_{2}, R_{1}, D\right\}$, Algorithm 1 may be deemed to have converged and the route $S-R_{2}-R_{1}-D$ is deemed to be the optimal route for transmission from $S$ to $D$.

The computational complexity has three main contributing factors: a) the calculation of a single NEC in a specific case; b) the number of NEC calculations; c) and finally, finding the minimum NEC in each round. Let us denote the complexity of $E_{s}$ in Eq. (31), $E_{f}$ in Eq. (32) and $p_{s}$, where $p_{s}=\prod_{1}^{H} B\left(p_{i}\right)$, by $\mathcal{C}\left(E_{s}\right), \mathcal{C}\left(E_{f}\right)$ and $\mathcal{C}\left(p_{s}\right)$. The complexity of evaluating $D_{s}$ and $D_{f}$ is the same as that of $E_{s}$ and $E_{f}$, apart from a multiplicative constant. The number of NEC calculations is given by the number of node pairs, which is $\mathcal{V}(\mathcal{V}-1) / 2$ (see line 5 and line 7 in Algorithm 1). The minimum NEC can be found based on the Fibonacci heap approach of [30], which has a complexity on the order of $\mathrm{O}(\log \mathcal{V})$. Therefore, the complexity imposed by Algorithm 1 is $\mathrm{O}\left[\mathcal{V}^{2}\left[\mathcal{C}\left(E_{s}\right)+\mathcal{C}\left(E_{f}\right)+\mathcal{C}\left(p_{s}\right)\right]+\mathcal{V} \log \mathcal{V}\right]$.

\section{B. OR algorithm}

In Section II-C, the minimum NEC is obtained by finding the optimal power allocation. Although the network topology in Fig. 2 has only two hops, this algorithm may be extended to a large network, where the OR principle is employed for each hop. Meanwhile, the optimal transmit power of each node is found for the sake of minimizing the NEC required for the successful passage of a packet from that node to the destination. Therefore, Algorithm 2 is conceived for calculating the minimum NEC by carrying out optimum distance-dependent power allocation at each node, hop-byhop. As a by-product, the delay distribution $\mathbf{D}_{s}(S)$ of a packet being successfully delivered to the destination and the delay distribution $\mathbf{D}_{f}(S)$ of a packet being dropped before reaching the destination are also delivered by Algorithm 2, where $\mathbf{D}_{s}(S)$ is the end-to-end delay distribution, which allows us to calculate the average end-to-end delay [31]. Moreover, the end-to-end throughput may also be calculated based on $\mathbf{D}_{s}(S), \mathbf{D}_{f}(S)$ and $p_{f}(S)$. Here, for any node $v$ in a given vertex set $\mathcal{V}, \bar{E}(v), \bar{E}(v, D)$ denotes the NEC $\bar{E}_{\text {total }}$ necessitated for transmission from node $v$ to the destination $D$, where we denote the potential set of receiver nodes by $\mathcal{R}$. Furthermore, $P_{t}^{o p t}(v)$ is the optimal transmit power, which minimizes the NEC required for transmission from node $v$ to the destination $D$, while $\mathbf{D}_{s}(v), \mathbf{D}_{s}(v, D)$ denotes the vector of probabilities for each particular integer delay in terms of TSs, when a packet is delivered successfully from node $v$ to the destination $D$. For example, a single-hop route has the vector of delay probabilities given by $\mathbf{D}_{s}(S, D)$, where the number of elements is $N_{r}$. The elements of $\mathbf{D}_{s}(S, D)$ are given by $\left(1-p_{s}\right)^{n_{r}} p_{s}$, where $p_{S}$ is the probability of a packet being successfully delivered to the destination $D$ and $n_{r}=1, \ldots, N_{r}$ is the number of MAC retransmissions. Furthermore, $\mathbf{D}_{s}^{n_{r}}(v)$ denotes the delay probability vector of a packet, which is delivered from node $v$ to the destination $D$, when the number of MAC retransmissions is $n_{r}$. By contrast, $\mathbf{D}_{f}(v), \mathbf{D}_{f}(v, D)$ denotes the delay probability vector of a packet, which is dropped before reaching the destination $D$. Let us denote the probability of a packet being successfully delivered from node $v$ to node $n$ by $p_{s}(v, n)$ and the probability of a packet, which is dropped before reaching the destination by $p_{f}(v), p_{f}(v, D)$. Moreover, $\gg n_{r}$ implies that the vector elements are shifted to the right by $n_{r}$ positions and the left positions are filled with zeros.

Lines 1-3 initialize the NEC between each node and the destination. Line 4 shows that $D$ is incorporated into the receiver set $\mathcal{R}$ and the probability vectors of delay $D_{s}(D)$ and $D_{f}(D)$ from $D$ to itself are set to unity. Initially, only $D$ belongs to $\mathcal{R}$ and $D$ has a neighbor node set. In line 8 , the notation $u$ represents the specific node, where $u \notin \mathcal{R}$ belongs to the neighbor set of $D$ at each loop. The optimal transmit power $P_{t}^{o p t}(u)$ of node $u$ can be obtained by setting the derivative of Eq. (54) with respect to $P_{t_{u}}$ to zero, which minimizes $\bar{E}(u, D)$. Meanwhile, the probability $p_{f}(u, D)$ is calculated by Eq. (53). Lines 23-25 show that if the NEC $\bar{E}(u, D)$ from node $u$ to $D$ via the receiver set $\mathcal{R}$ is lower than the stored NEC $\bar{E}(u)$, then $\bar{E}(u)$, the probability vector of delay $D_{s}(u), D_{f}(u)$ and the delivery failure probability 


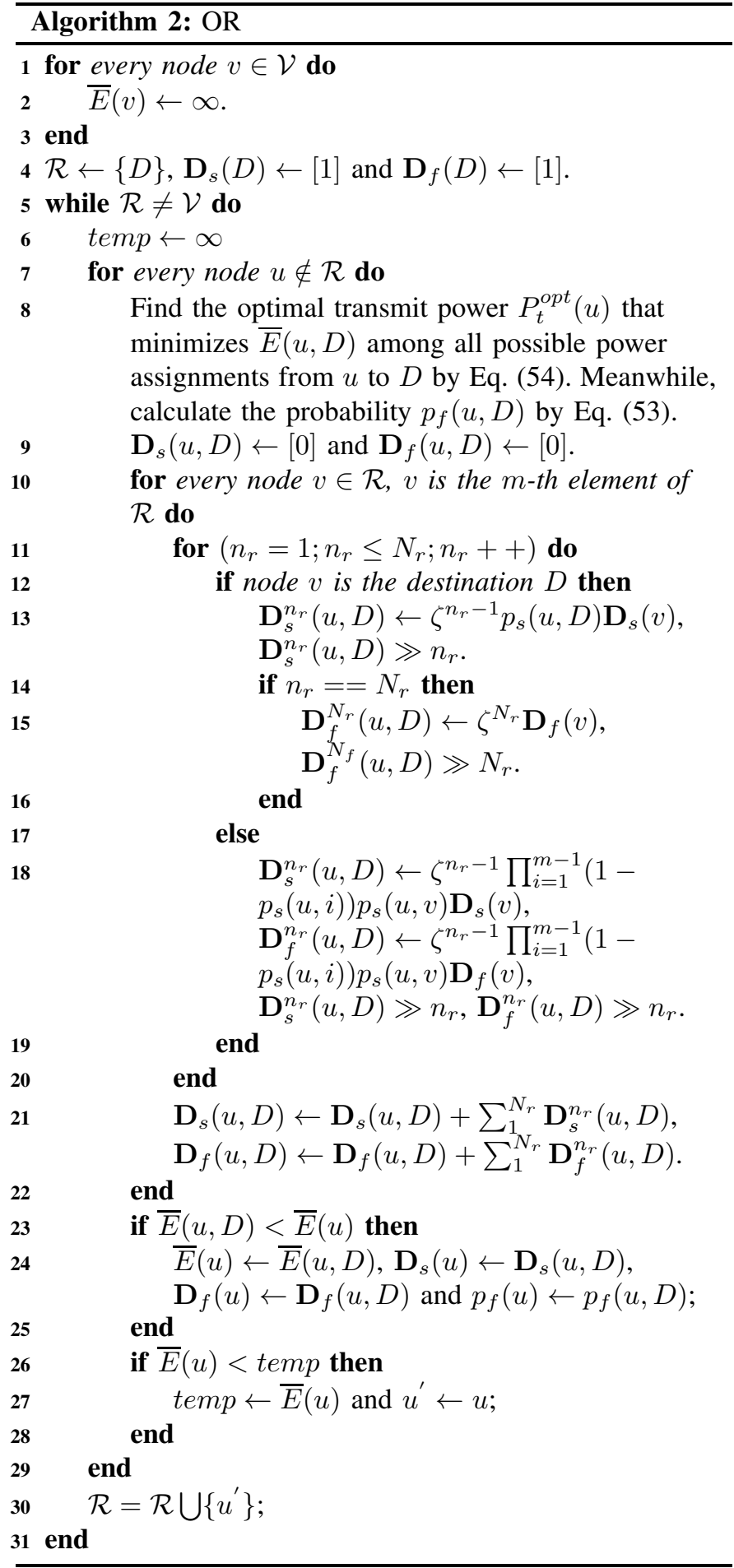

$p_{f}(u)$ are updated. Lines 26-28 show that if a node $u$ has the lowest NEC $\bar{E}(u, D)$ in each loop, then it will be represented as $u^{\prime}$ and be incorporated into $\mathcal{R}$, as seen in line 30. Again, in the next loop, any node, which belongs to the neighbor set of $u^{\prime}$ and does not belong to $\mathcal{R}$, will become a new $u$. The new $u$ and $\mathcal{R}$ will contain a new opportunistic relaying subnetwork, where the new sub-network topology is similar as the one in Fig. 2 and the optimal transmit power of each relay has already been obtained in the previous loops. Algorithm 2 would converge, when we have $\mathcal{R}=\mathcal{V}$, as seen in line 5. Like TR, Algorithm 2 is also a locally optimal algorithm, which is in fact optimal for every single hop. This can be also explained by the calculation of the optimal transmit power $P_{t}^{o p t}(u)$ in line 8.

Moreover, lines 9-22 detail how to generate the delay distribution for the path spanning from node $u$ to $D$. In each loop, node $u$ and the receiver set $\mathcal{R}$ constitute a subnetwork. The delay distribution of every node $v$ in $\mathcal{R}$ has been obtained in the previous loops. As described above, the delay distribution of node $v$ is a $N_{r}$-element vector $D_{s}(v)$, where the value of each element is the probability of a particular integer delay quantified in terms of the number of TSs. Lines 13-16 calculate the delay distribution $D_{s}(u, D)$ if $D$ receives the packet from $u$, while line 18 calculates the delay distribution $D_{s}(u, D)$ if node $v$ in $\mathcal{R}$ - except for $D$ - receives the packet from $u$. The for loop in line 11 considers all conditions, when the number of MAC retransmissions from $u$ to $\mathcal{R}$ is $1 \leq N_{r} \leq N_{r}$, where the sum is calculated in line 21. Finally, when Algorithm 2 converges, we obtain the end-to-end delay distribution, which is $D_{s}(S)$.

Fig. 4 shows the step-by-step execution of Algorithm 2 using the NEC metric $\bar{E}_{\text {total }}$. Both the topology and the relevant parameters are similar to those used for TR. We also assumed that each node is aware of the other nodes' position, hence also of their distance. In a compact form, we have $\mathcal{V}=\left\{S, R_{1}, R_{2}, D\right\}$ and $\mathcal{R}=\{D\}$, as shown in Fig. 4(a). In Fig. 4(b), $S, R_{1}$ and $R_{2}$ calculate their transmit power optimized for minimizing the NEC from Eq. (54), yielding $\bar{E}_{S D}=3.4 \times 10^{-8} \mathrm{~mJ} / \mathrm{bit}, \bar{E}_{R_{1} D}=1.7 \times 10^{-8} \mathrm{~mJ} / \mathrm{bit}$, $\bar{E}_{R_{2} D}=2.4 \times 10^{-8} \mathrm{~mJ} / \mathrm{bit}$ for transmission to $D$, as also seen in line 8 of Algorithm 2. Since $\bar{E}_{R_{1} D}=1.7 \times 10^{-8} \mathrm{~mJ} / \mathrm{bit}$ is the lowest in the set of the three energies, we update $\mathcal{R}$ to $\left\{R_{1}, D\right\}$, as shown in line 26 and line 30 of Algorithm 2. Then in Fig. 4(c), $S$ and $R_{2}$ adjust their own transmit power and update their NEC for transmission to node $D$ by considering $\left\{R_{1}, D\right\}$ as their forwarder relay set, as shown in line 23 of Algorithm 2. Since $\bar{E}_{R_{2} D}=2.0 \times 10^{-8} \mathrm{~mJ} / \mathrm{bit}$ is lower than $\bar{E}_{S D}=2.5 \times 10^{-8} \mathrm{~mJ} / \mathrm{bit}, \mathcal{R}$ is updated to $\left\{R_{2}, R_{1}, D\right\}$, as shown in line 26 and line 30 of Algorithm 2. Finally, in Fig. 4(d), $S$ adjusts its own transmit power to the optimal one, which minimizes $\bar{E}_{S D}=2.3 \times 10^{-8} \mathrm{~mJ} / \mathrm{bit}$, where $\left\{R_{2}, R_{1}, D\right\}$ is the resultant forwarder relay set, as shown in line 8 of Algorithm 2. At this stage, Algorithm 2 may be deemed to have converged, since $S$ is incorporated into $\mathcal{R}$ and we have $\mathcal{R}=\left\{S, R_{2}, R_{1}, D\right\}$. In this algorithm, every node has to find its own forwarder R-set by itself upon exploiting the knowledge of the other nodes' positions. If more than one node in a node's forwarder R-list receives the packet from that node successfully, then that particular one, which requires the lowest NEC for transmission to the destination has the highest priority for forwarding this packet. The nodes of the forwarder R-set communicate with each other similarly to the technique of [27] and again, the NEC required for successful transmission to $D$ is invoked for deciding the priority order of the forwarders.

The complexity of finding the transmit power and the forwarder set also depends on three contributing factors, just like for the TR scenario. In Algorithm 2, the delay distribution D does not affect the complexity of finding the transmit power and the forwarder set. Let us denote the complexity of $E_{\text {total }}$ 


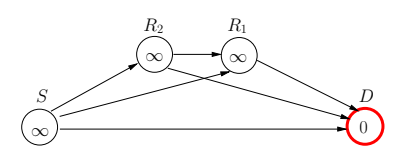

(a)
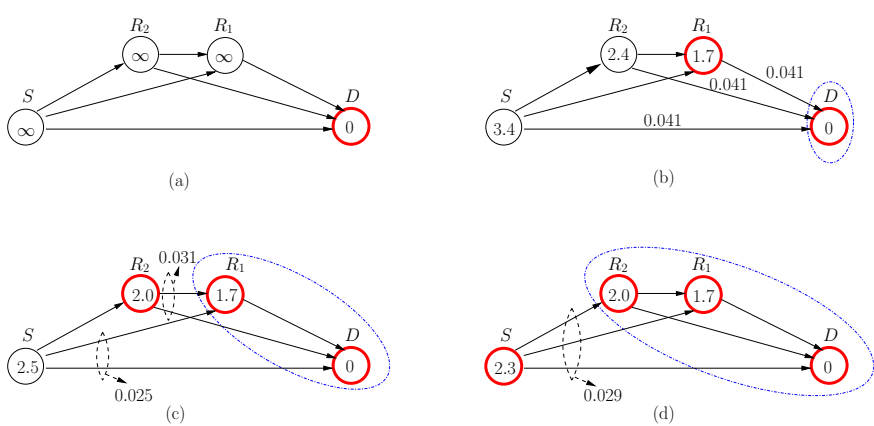

Fig. 4. Execution of Algorithm 2. The value within a node $u$ is its cost $\bar{E}_{\text {total }}\left(\times 10^{-8} \mathrm{~mJ} / \mathrm{bit}\right)$ incurred by its transmission from node $u$ to $D$ and the dash-dot ellipse represents the receiver set $\mathcal{R}$ before each iteration. After each iteration one node is incorporated into the set $\mathcal{R}$. The nodes in boldface denote the nodes in $\mathcal{R}$ after each iteration. The values next to the arrows or the dashed ellipses represent the probability $p_{f}(u, \mathcal{R})$ of a packet being transmitted from $S$ in conjunction with the event that none of the nodes in the receiver set $\mathcal{R}$ receives it after $N_{r}=7$ retransmissions. (a) The situation just after the initialization, where $\mathcal{R}=\{D\}$. (b) The first iteration of the algorithm, where we have $\mathcal{R}=\{D\}$ before the iteration, while after the iteration $R_{1}$ is incorporated into the set $\mathcal{R}$ with the optimum power of $P_{t}^{\text {opt }}\left(R_{1}\right)=0.39 \mathrm{~mW}$, yielding $\mathcal{R}=\left\{R_{1}, D\right\}$. (c) The second iteration of the algorithm, where we have $\mathcal{R}=\left\{R_{1}, D\right\}$ before the iteration while after the iteration $R_{2}$ is incorporated into the set $\mathcal{R}$ with the optimum power of $P_{t}^{\text {opt }}\left(R_{2}\right)=0.36 \mathrm{~mW}$, yielding $\mathcal{R}=\left\{R_{2}, R_{1}, D\right\}$. (d) The final iteration of algorithm, where we have $\mathcal{R}=\left\{R_{2}, R_{1}, D\right\}$ before the iteration, while after the iteration $S$ is incorporated into the set $\mathcal{R}$ with the optimum power of $P_{t}^{o p t}(S)=0.41 \mathrm{~mW}$, yielding $\mathcal{R}=\left\{S, R_{2}, R_{1}, D\right\}$. The algorithm terminates.

in Eq. (47) and of $p_{f}$ in Eq. (53) by $\mathcal{C}\left(E_{\text {total }}\right)$ and $\mathcal{C}\left(p_{f}\right)$, respectively. Then line 5 in Algorithm 2 has to be invoked $\mathcal{V}$ times, adding a further node into $\mathcal{R}$ in each round (in line 30 of Algorithm 2). Line 7 suggests that the optimal transmit power of any node in $(\mathcal{V}-\mathcal{R})$ is calculated in a specific round and the complexity of this calculation is given by $\mathcal{C}\left(E_{\text {total }}\right)+\mathcal{C}\left(p_{f}\right)$. Again, the complexity of finding the optimal transmit power can be calculated by Fibonacci heap [30] which has a complexity on the order of $\mathrm{O}(\log \mathcal{V})$. Therefore, the complexity of Algorithm 2 is $\mathrm{O}\left[\mathcal{V}^{2}\left[\mathcal{C}\left(E_{\text {total }}\right)+\mathcal{C}\left(p_{f}\right)\right]+\mathcal{V} \log \mathcal{V}\right]$

\section{Performance Study}

Having defined the energy-consumption-based OF and the optimal transmit power of both the TR and of the OR, let us now characterize the attainable system performance in terms of:

- Normalized energy consumption (mJ/bit): The total energy consumed by all the data packets transmitted through the network is normalized by the successful end-to-end reception probability. However, we do not consider the energy consumed by the control packets and the energy dissipated at the receiver nodes by signal processing.

- End-to-end throughput (bit/s): The number of data bits successfully delivered to the destination in a second, while neglecting the bits of the control packets.

- delay PDF: The probability distribution of the end-to-end delay.

The common parameters of the following simulations are listed in Tab I.
TABLE I

THE COMMON SIMULATION CONFIGURATION

\begin{tabular}{|l|l|}
\hline Packet Length & 8688 bits \\
\hline coding \& modulation & IrCC-URC-QPSK \\
\hline Channel model & $\begin{array}{l}\text { uncorrelated and } \\
\text { non-dispersive Rayleigh channel }\end{array}$ \\
\hline Path-loss exponent & 2 \\
\hline Information transmission rate & $6 \mathrm{Mbits} / \mathrm{s}$ \\
\hline Thermal noise power & $-110 \mathrm{dBm}$ \\
\hline
\end{tabular}

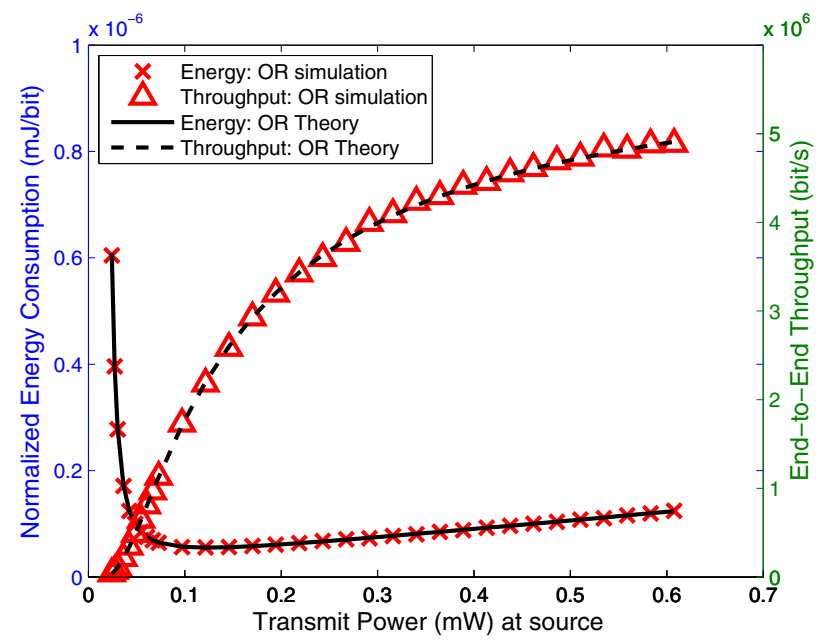

Fig. 5. The NEC $\bar{E}_{\text {total }}$ and the end-to-end throughput $R_{e 2 e}$ versus the transmit power $P_{t_{S}}$.

Example 1: Let us first analyze the performance of a singlehop route. The maximum number of MAC retransmissions is $N_{r}=7$. The distance between $S$ and $D$ is $1000 \mathrm{~m}$. The other simulation configurations are listed in Tab I. The NEC $\bar{E}_{\text {total }}$ and the end-to-end throughput $R_{e 2 e}$ of the TR and the OR are compared both in terms of simulation and theoretical results in Fig. 5.

Fig. 5 shows that the NEC initially decreases and then increases slowly beyond the transmit power of $0.12 \mathrm{~mW}$, where $0.12 \mathrm{~mW}$ is the optimal transmit power obtained by using Eq. (17). The end-to-end throughput increases upon increasing the transmit power at $S$. Observe that the simulation results closely match the theoretical curve.

Example 2: Let us now analyze the performance of the networks associated with a total of $N=4$ and 15 nodes. The positions of $S$ and $D$ are $(100,100)$ and $(900,100)$, respectively, while the other nodes are uniformly located within a circle centered at $(400,100)$ with a radius of $400 \mathrm{~m}$. The NEC $\bar{E}_{\text {total }}$ and the end-to-end throughput $R_{e 2 e}$ are shown in Fig. 6 and Fig. 7 as a function of the maximum number of MAC retransmissions $N_{r}$. We also investigated the theoretical NEC bound of both TR and OR when $N=4$, which was found by the exhaustive search of all the routes spanning from $S$ to $D$, while for $N=15$ no theoretical bounds were given, since the exhaustive search has an excessive computation complexity. Fig. 6 shows that the performance of the energy-consumption $\mathrm{OF}$ based algorithm is close to the theoretical bound when $N=4$, especially in the case of a high $N_{r}$. Both Figs. 6 and 7 show that our energy-efficient OR outperforms both the Adjustable Energy-Efficient Opportunistic Routing (A- 
EEOR) algorithm defined in [3] and the energy-efficient TR. Compared to the A-EEOR algorithm our OR algorithm has a lower normalized energy consumption for $N_{r}<4$, as seen in Fig. 6, while exhibiting a higher end-to-end throughput for $N_{r}<6$, as shown in Fig. 7. Moreover, both our OR and TR simulation results closely match the theoretical curves. When we have $N_{r}=1$ or 2 for the network topology of $N=4$, both the exhaustive search, labelled by "TR bound" and our proposed TR algorithm, labelled by "TR theory", selected the route 'S-D', hence, the NEC is the same for both. When we have $2<N_{r}<8$, the exhaustive search and our proposed TR algorithm choose different routes, since the exhaustive search represents the globally optimal algorithm, while our proposed TR algorithm is a locally optimal algorithm. More specifically, our TR algorithm is optimal for every single hop. Moreover, the simulation results corresponding to the 'TR simulation' label closely match the theoretical value represented by the label 'TR theory'. Therefore, the 'TR simulation/theory' and the 'TR bound' scenarios exhibit a performance discrepancy, when we have $2<N_{r}<8$, as seen in Fig. 6 . Note that the NEC $\bar{E}_{\text {total }}$ decreases upon increasing $N_{r}$. However, the end-to-end throughput $R_{e 2 e}$ of the A-EEOR and OR regimes first increases and then saturates. Additionally, the end-to-end throughput of TR is in fact higher than that of OR for $N_{r}=1$ and 2 when $N=4$, but it is lower for $N_{r} \geq 3$, as seen in Fig. 7. This is because in case of a low number of MAC retransmissions, the direct near-line-of-sight route spanning from $S$ to $D$ in the TR has a more dominant priority than the other routes.

We then continued by investigating the delay-PDF of the system in Fig. 8 versus the end-to-end delay $D_{e 2 e}$, which was quantified in terms of the number of transmit TSs. Each TS has a duration of $T$ and a packet is forwarded by a node during $T$. Fig. 8 shows that the proposed energy-efficient OR algorithm has a lower end-to-end delay than the TR algorithm, since the delay PDF curve of the OR algorithm reaches its peak probability at the delay of about 2 TSs when $N=4$ and 6 TSs when $N=15$, while that of the TR algorithm at 6 TSs when $N=4$ and 14 TSs when $N=15$.

Fig. 9 illustrates that the optimal transmit power set $\mathcal{P}$ found by our proposed energy-efficient OR algorithm is close the optimal one generated by the exhaustive search when $N=4$. The optimal transmit group $\mathcal{P}$ is defined as the optimal power set of the node $S, R_{1}$ and $R_{2}$, which is $\left\{P_{S_{\text {opt }}}, P_{R_{1} \text { opt }}, P_{R_{2 o p t}}\right\}$. If we increase or decrease the transmit power by a sub-optimum multiplicative factor $k$ yielding $\left\{k P_{S_{\text {opt }}}, k P_{R_{1} \text { opt }}, k P_{R_{2 o p t}}\right\}$, then the NEC $\bar{E}_{\text {total }}$ becomes higher than that associated with the optimal transmit power, as seen in Fig. 9. The dashed line in Fig. 9 indicates the theoretical minimum NEC $\bar{E}_{\text {total }}$ for the TR algorithm, which should be a single point in the figure for $k=1$. However, we extend this point to a dashed line to show the advantage of our OR algorithm. As seen in Fig. 9, the curve characterizing the OR algorithm is below the curve of the TR algorithm for $0.6<k<2.2$, which means that our OR algorithm still outperforms the TR algorithm, although the OR does not achieve its own best performance. The OR algorithm improves the NEC by $15.57 \%$ compared to the TR algorithm at the optimal point for $k=1$.

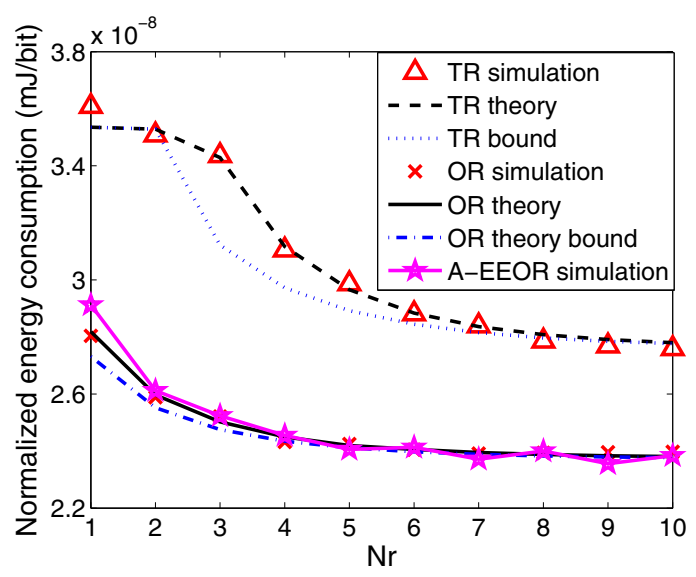

(a) Network topology $N=4$.

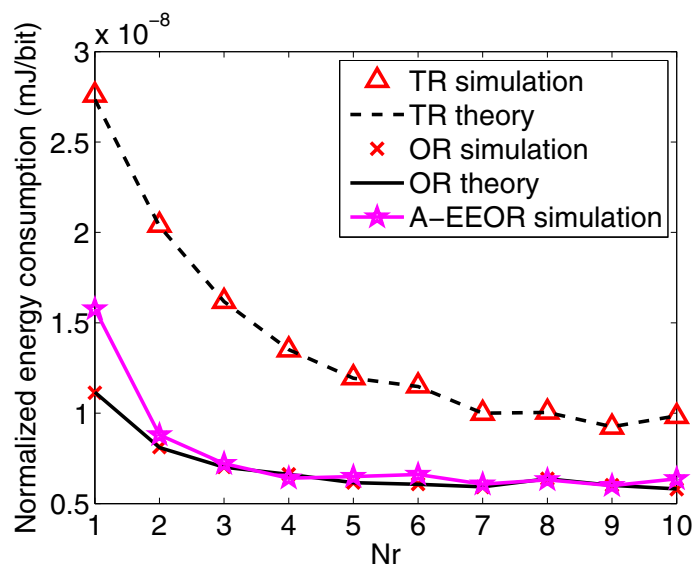

(b) Network topology $N=15$.

Fig. 6. The NEC $\bar{E}_{\text {total }}$ versus the maximum number of MAC retransmissions $N_{r}$ when $N=4$ and 15 .

\section{Conclusions}

This paper proposed a cross-layer operation aided energyefficient OR algorithm for Ad Hoc networks and an energyconsumption-based OF combined with power allocation, which is employed both for finding a theoretical bound and for conveying the packets through the network. The energyconsumption-based $\mathrm{OF}$ and the related algorithms are applicable to both traditional routing and to our opportunistic routing. They also perform close to the respective bounds found by an exhaustive search and match the simulation results quite closely. The end-to-end throughput, the end-to-end delay and the delay distribution of the system were also analyzed theoretically. In conclusion, the OR outperforms the TR.

\section{REFERENCES}

[1] X.-Y. Li, Y. Wang, H. Chen, X. Chu, Y. Wu, and Y. Qi, "Reliable and energy-efficient routing for static wireless ad hoc networks with unreliable links," IEEE Trans. Parallel and Distrib. Syst., vol. 20, no. 10, pp. 1408-1421, Oct. 2009.

[2] M. Zorzi and R. R. Rao, "Geographic random forwarding (GeRaF) for ad hoc and sensor networks: energy and latency performance," IEEE Trans. Mobile Comput., vol. 2, no. 4, pp. 349-365, Oct.-Dec. 2003.

[3] X. Mao, S. Tang, X. Xu, X.-Y. Li, and H. Ma, "Energy-efficient opportunistic routing in wireless sensor networks," IEEE Trans. Parallel and Distrib. Syst., vol. 22, no. 11, pp. 1934-1942, Nov. 2011.

[4] M. Dehghan, M. Ghaderi, and D. Goeckel, "Minimum-energy cooperative routing in wireless networks with channel variations," IEEE Trans. Wireless Commun., vol. 10, no. 11, pp. 3813-3823, Nov. 2011. 


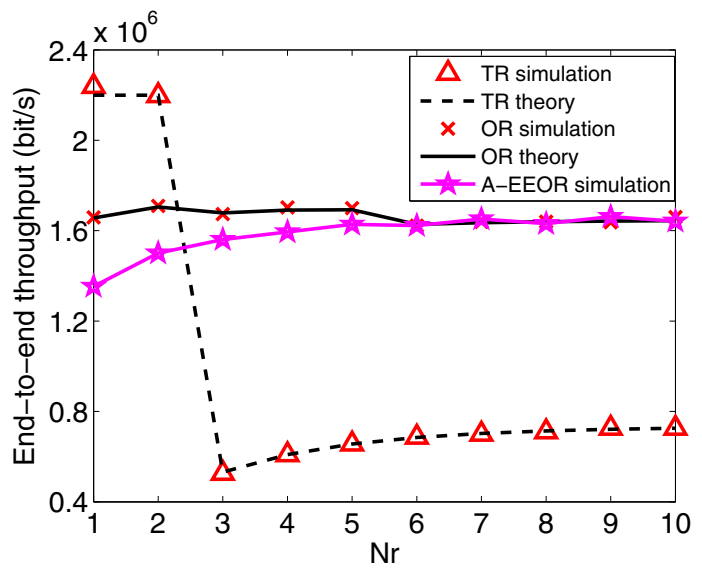

(a) Network topology $N=4$.

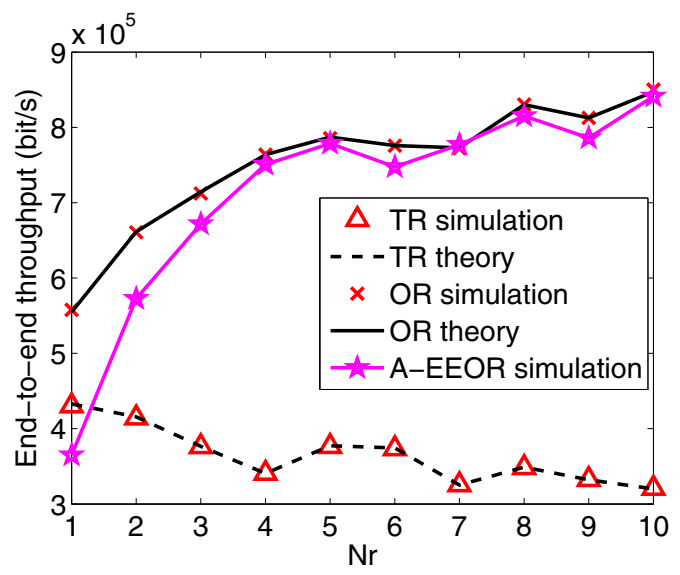

(b) Network topology $N=15$.

Fig. 7. The end-to-end throughput $R_{e 2 e}$ versus the maximum number of MAC retransmissions $N_{r}$ when $N=4$ and 15 .

[5] J. Zhu and X. Wang, "Model and protocol for energy-efficient routing over mobile ad hoc networks," IEEE Trans. Mobile Comput., vol. 10, no. 11, pp. 1546-1557, Nov. 2011.

[6] T. Luo, M. Motani, and V. Srinivasan, "Energy-efficient strategies for cooperative multichannel MAC protocols," IEEE Trans. Mob. Comput., vol. 11, no. 4, pp. 553-566, Apr. 2012.

[7] S. Kwon and N. Shroff, "Energy-efficient SINR-based routing for multihop wireless networks," IEEE Trans. Mob. Comput., vol. 8, no. 5, pp. 668-681, May 2009.

[8] C. Wei, C. Zhi, P. Fan, and K. B. Letaief, "AsOR: an energy efficient multi-hop opportunistic routing protocol for wireless sensor networks over Rayleigh fading channels," IEEE Trans. Wireless Commun., vol. 8, no. 5, pp. 2452-2463, May 2009.

[9] M. C. Vuran and I. F. Akyildiz, "XLP: a cross-layer protocol for efficient communication in wireless sensor networks," IEEE Trans. Mob. Comput., vol. 9, no. 11, pp. 1578-1591, Nov. 2010.

[10] H. Kwon, T. H. Kim, S. Choi, and B. G. Lee, "A cross-layer strategy for energy-efficient reliable delivery in wireless sensor networks," IEEE Trans. Wireless Commun., vol. 5, no. 12, pp. 3689-3699, Dec. 2006.

[11] S. Biswas and R. Morris, "Opportunistic routing in multi-hop wireless networks," ACM SIGCOMM Computer Commun. Rev., vol. 34, no. 1, pp. 69-74, Jan. 2004.

[12] Q. W. Liu, S. L. Zhou, and G. B. Giannakis, "Cross-layer combining of adaptive modulation and coding with truncated ARQ over wireless links," IEEE Trans. Wireless Commun., vol. 3, no. 5, pp. 1746-1755, Sept. 2004.

[13] Z. Wang, Y. Chen, and C. Li, "CORMAN: a novel cooperative opportunistic routing scheme in mobile ad hoc networks," IEEE J. Sel. Areas Commun., vol. 30, no. 2, pp. 289-296, Feb. 2012.

[14] C. E. Perkins, E. M. Royer, S. R. Das, and M. K. Marina, "Performance comparison of two on-demand routing protocols for ad hoc networks," IEEE Personal Commun., vol. 8, no. 1, pp. 16-28, Feb. 2001.

[15] M. Zorzi and R. R. Rao, "Geographic random forwarding (GeRaF) for

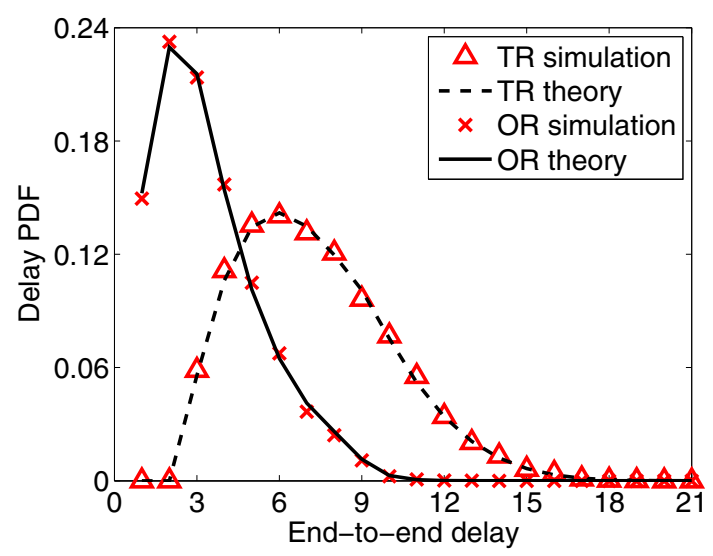

(a) Network topology $N=4$.

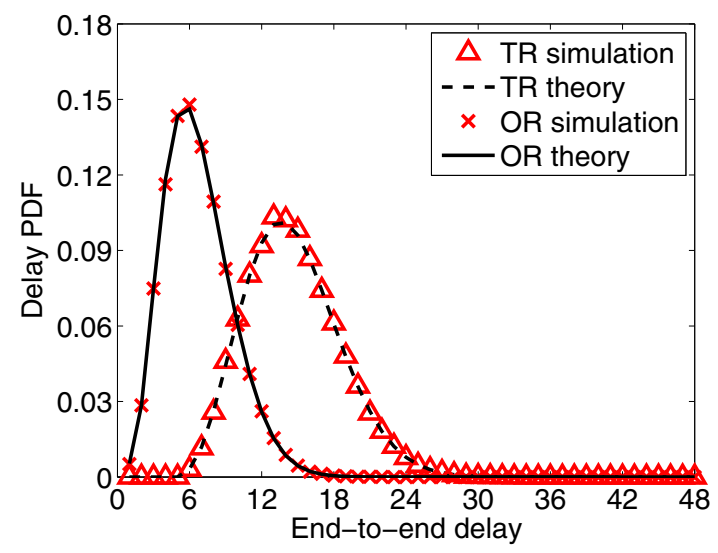

(b) Network topology $N=15$.

Fig. 8. The delay distribution when $N=4$ and 15 .

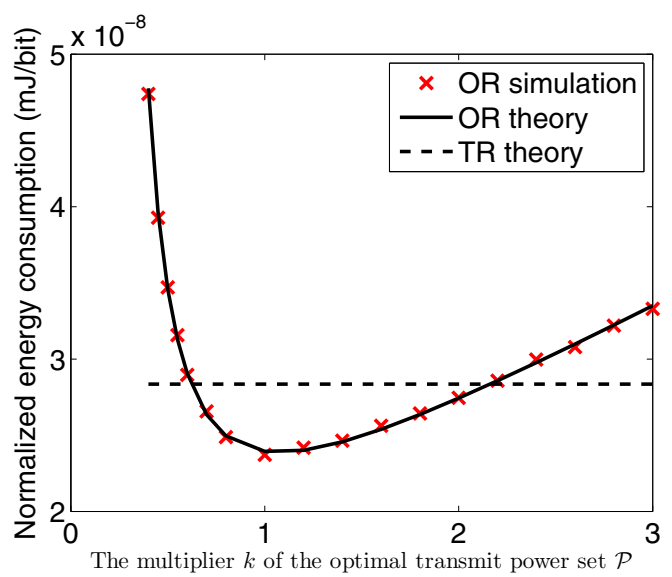

Fig. 9. The NEC $\bar{E}_{\text {total }}$ versus the multiplier $k$ of the optimal transmit power set $\mathcal{P}$.

ad hoc and sensor networks: multihop performance," IEEE Trans. Mob. Comput., vol. 2, no. 4, pp. 337-348, Oct.-Dec. 2003.

[16] H. Liu, B. Zhang, H. Mouftah, X. Shen, and J. Ma, "Opportunistic routing for wireless ad hoc and sensor networks: present and future directions," IEEE Commun. Mag., vol. 47, no. 12, pp. 103-109, Dec. 2009.

[17] H. Dubois-Ferrière, M. Grossglauser, and M. Vetterli, "Valuable detours: least-cost anypath routing," IEEE/ACM Trans. Networking, vol. 19, no. 2, pp. 333-346, Apr. 2011.

[18] A. A. Bhorkar, M. Naghshvar, T. Javidi, and B. D. Rao, "Adaptive opportunistic routing for wireless ad hoc networks," IEEE/ACM Trans. Networking, vol. 20, no. 1, pp. 243-256, Feb. 2012.

[19] K. Zeng, Z. Yang, and W. Lou, "Location-aided opportunistic forwarding 
in multirate and multihop wireless networks," IEEE Trans. Veh. Technol., vol. 58, no. 6, pp. 3032-3040, July 2009.

[20] R. Laufer, H. Dubois-Ferrière, and L. Kleinrock, "Polynomial-time algorithms for multirate anypath routing in wireless multihop networks," IEEE/ACM Trans. Networking, vol. 20, no. 3, pp. 742-755, June 2012.

[21] T. H. Cormen, C. E. Leiserson, R. L. Rivest, and C. Stein, Introduction to Algorithms, 3rd ed. MIT Press, 2009.

[22] J. Zuo, C. Dong, S. X. Ng, L.-L. Yang, and L. Hanzo, "Energy-efficient routing in ad hoc networks relying on channel state information and limited MAC retransmissions," in 2011 IEEE Vehicular Technology Conference - Fall.

[23] J. Zuo, H. V. Nguyen, S. X. Ng, and L. Hanzo, "Energy-efficient relay aided ad hoc networks using iteratively detected irregular convolutional coded, unity-rate coded and space-time trellis coded transceivers," in 2011 IEEE Wireless Communications and Networking Conference.

[24] H. V. Nguyen, S. X. Ng, J. L. Rebelatto, Y. Li, and L. Hanzo, "Nearcapacity network coding for cooperative multi-user communications," in 2011 IEEE Vehicular Technology Conference - Fall.

[25] I. Gradshteyn and I. Ryzhik, Table of Integrals, Series, and Products, 7th edition. Elsevier Pte Ltd., 2007.

[26] Available: http://functions.wolfram.com/

[27] C. Dong, L.-L. Yang, and L. Hanzo, "Performance analysis of multi-hop diversity aided multi-hop links over Nakagami-m fading channels with adaptive modulation." Available: http://eprints.soton.ac.uk/343477/

[28] — "Performance analysis of multihop-diversity-aided multihop links," IEEE Trans. Veh. Technol., vol. 61, no. 6, pp. 2504-2516, July 2012.

[29] E. W. Dijkstra, "A note on two problems in connexion with graphs," Numerische Mathematik, vol. 1, no. 1, pp. 269-271, 1959.

[30] M. L. Fredman and R. E. Tarjan, "Fibonacci heaps and their uses in improved network optimization algorithms," J. Association for Computing Machinery, vol. 34, no. 3, pp. 596-615, July 1987.

[31] C. Dong, L.-L. Yang, and L. Hanzo, "Performance of buffer-aided adaptive modulation in multihop communications." Available: http: //eprints.soton.ac.uk/348686/

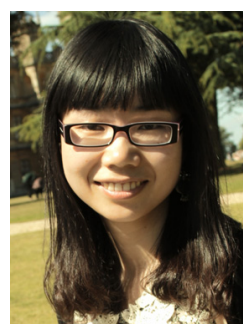

Jing Zuo (S'09) received her BEng degree in Communications Engineering and her MSc degree in Communications and Information System from Jilin University, Changchun, China, in 2006 and 2008; and her Ph.D. degree in Wireless Communications from University of Southampton, U.K., in 2013. She is the recipient of scholarship under the UK-China Scholarships for Excellence programme from 2008 to 2011. From 2009 to 2013, she was involved in the OPTIMIX and CONCERTO European projects. Her current research interests include protocols and algorithms design, cross-layer optimization and opportunistic communications.

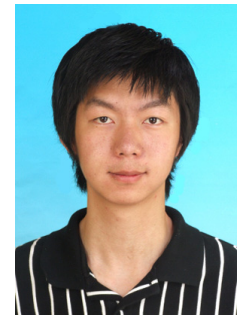

Chen Dong received his BS degree in electronic information sciences and technology from University of Science and Technology of China(USTC), Hefei, China in 2004, and his MEng degree in pattern recognition and automatic equipment from the Graduate University of Chinese Academy of Sciences(GUCAS), Beijing, China in 2007. Since 2009, he has been working towards his Ph.D. degree with the Communications, Signal Processing and Control (CSPC) group, School of Electronics and Computer Science, University of Southampton, UK. $\mathrm{He}$ is the recipient of scholarship under the UK-China Scholarships for Excellence programme. His research interests include applied math, relay system, channel modelling and cross-layer optimization.

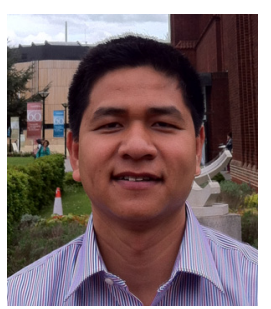

Hung Viet Nguyen received the B.Eng. degree in Electronics \& Telecommunications from Hanoi University of Science and Technology (HUST), Hanoi, Vietnam, in 1999, the M.Eng. in Telecommunications from Asian Institute of Technology (AIT), Bangkok, Thailand, in 2002 and the Ph.D. degree in wireless communications from the University of Southampton, Southampton, U.K., in 2013. Since 1999 he has been a lecturer at the Post \& Telecommunications Institute of Technology (PTIT), Vietnam. His research interests include cooperative communications, channel coding and network coding.

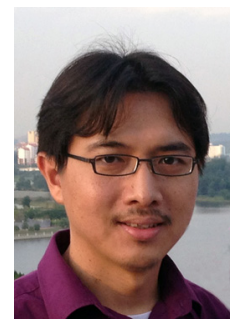

Soon Xin Ng (S'99-M'03-SM'08) received the B.Eng. degree (First class) in electronics engineering and the Ph.D. degree in wireless communications from the University of Southampton, Southampton, U.K., in 1999 and 2002, respectively. From 2003 to 2006, he was a postdoctoral research fellow working on collaborative European research projects known as SCOUT, NEWCOM and PHOENIX. Since August 2006, he has been a member of academic staff in the School of Electronics and Computer Science, University of Southampton. He is involved in the OPTIMIX and CONCERTO European projects as well as the IU-ATC and UC4G projects. He is currently a senior lecturer at the University of Southampton.

His research interests include adaptive coded modulation, coded modulation, channel coding, space-time coding, joint source and channel coding, iterative detection, OFDM, MIMO, cooperative communications, distributed coding, quantum error correction codes and joint wireless-and-optical-fiber communications. He has published over 160 papers and co-authored two John Wiley/IEEE Press books in this field. He is a Senior Member of the IEEE, a Chartered Engineer and a Fellow of the Higher Education Academy in the UK.

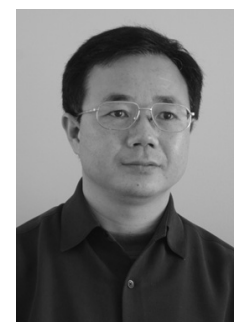

Lie-Liang Yang (M'98, SM'02) received his BEng degree in communications engineering from Shanghai TieDao University, Shanghai, China in 1988, and his MEng and Ph.D. degrees in communications and electronics from Northern (Beijing) Jiaotong University, Beijing, China in 1991 and 1997, respectively. From June 1997 to December 1997 he was a visiting scientist of the Institute of Radio Engineering and Electronics, Academy of Sciences of the Czech Republic. Since December 1997, he has been with the University of Southampton, United Kingdom, where he is the professor of wireless communications in the School of Electronics and Computer Science. Dr. Yang's research has covered a wide range of topics in wireless communications, networking and signal processing. He has published over 300 research papers in journals and conference proceedings, authored/co-authored three books and also published several book chapters. The details about his publications can be found at http://www-mobile.ecs.soton.ac.uk/lly/. He is a fellow of the IET, served as an associate editor to the IEEE Trans. on Vehicular Technology and Journal of Communications and Networks (JCN), and is currently an associate editor to the IEEE Access and the Security and Communication Networks (SCN) Journal.

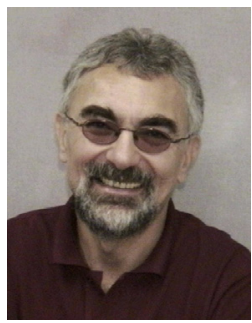

Lajos Hanzo (http://www-mobile.ecs.

soton.ac.uk) FREng, FIEEE, FIET, Fellow of EURASIP, DSc received his degree in electronics in 1976 and his doctorate in 1983. In 2009 he was awarded the honorary doctorate "Doctor Honoris Causa" by the Technical University of Budapest. During his 37-year career in telecommunications he has held various research and academic posts in Hungary, Germany and the UK. Since 1986 he has been with the School of Electronics and Computer Science, University of Southampton, UK, where he holds the chair in telecommunications. He has successfully supervised $80+$ $\mathrm{PhD}$ students, co-authored $20 \mathrm{John}$ Wiley/IEEE Press books on mobile radio communications totalling in excess of 10000 pages, published 1300+ research entries at IEEE Xplore, acted both as TPC and General Chair of IEEE conferences, presented keynote lectures and has been awarded a number of distinctions. Currently he is directing a 100-strong academic research team, working on a range of research projects in the field of wireless multimedia communications sponsored by industry, the Engineering and Physical Sciences Research Council (EPSRC) UK, the European Research Council's Advanced Fellow Grant and the Royal Society's Wolfson Research Merit Award. He is an enthusiastic supporter of industrial and academic liaison and he offers a range of industrial courses. He is also a Governor of the IEEE VTS. During 2008 - 2012 he was the Editor-in-Chief of the IEEE Press and a Chaired Professor also at Tsinghua University, Beijing. His research is funded by the European Research Council's Senior Research Fellow Grant. For further information on research in progress and associated publications please refer to http://www-mobile.ecs.soton.ac.uk Lajos has 18 000+ citations. 